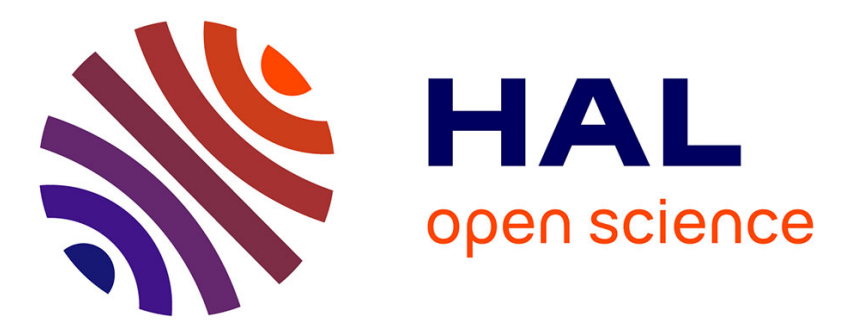

\title{
Nearest neighbor balanced block designs for autoregressive errors
}

Mamadou Koné, Annick Valibouze

\section{To cite this version:}

Mamadou Koné, Annick Valibouze. Nearest neighbor balanced block designs for autoregressive errors. Metrika, 2021, 84 (3), pp.281-312. 10.1007/s00184-020-00770-6 . hal-01850854v2

\section{HAL Id: hal-01850854 \\ https://hal.sorbonne-universite.fr/hal-01850854v2}

Submitted on 29 Sep 2018 (v2), last revised 8 Sep 2021 (v3)

HAL is a multi-disciplinary open access archive for the deposit and dissemination of scientific research documents, whether they are published or not. The documents may come from teaching and research institutions in France or abroad, or from public or private research centers.
L'archive ouverte pluridisciplinaire HAL, est destinée au dépôt et à la diffusion de documents scientifiques de niveau recherche, publiés ou non, émanant des établissements d'enseignement et de recherche français ou étrangers, des laboratoires publics ou privés. 


\title{
NEAREST NEIGHBOR BALANCED BLOCK DESIGNS FOR AUTOREGRESSIVE ERRORS AR( $m)$
}

\author{
MAMADOU KONÉ AND ANNICK VALIBOUZE
}

\begin{abstract}
The problem of finding neighbor optimal designs for a general correlation structure is studied. This paper gives optimality universal conditions for incomplete block designs when observations on the same block are modeled by an autoregressive process $\mathrm{AR}(m)$ of an arbitrary order $m$. It extends and generalizes existing results for models AR(1) and AR(2) as in such as those of Grondona and Cressie (1993) for AR(2) and those of Gill and Shukla (1985) and Kunert (1987) for AR(1).
\end{abstract}

Keywords: block design, autoregressive model, nearest-neighbor balanced, generalized least squares estimation, universally optimal.

\section{INTRODUCTION}

We owe the systematic introduction of statistical methods in the planning of experiments to the British Statistician R. A. Fisher. During the years 1925-1937, in the Rothamsted (GB) Agricultural Research Station, he intiated taking account in the results of experiments of the heterogenity of parcels (testing grounds) by comparing the output of different cropped varieties; he then introduced complete block designs, incomplete block designs and Latin squares.

The construction and use of these designs rely on three principles, namely, repetition, randomisation and local control. The objective of repetition is to enable an estimation of the residual variability and to increase the experiment precision. The randomisation allows to obtain unbiased estimations of the residual variability and of the influence of varieties on the performance. Like repetition, the aim of the local control is to increase the experiment precision.

Over the decades that followed this initial works, the principles developed by Fisher in agronomy have been transposed into various other sectors of activity such as industry and services sector Among the new concepts that have been introduced, we mention the

Date: September 29, 2018.

This research was partly supported by the Galois project. 
concept of optimal design due to J. Kieffer (1958-1981). At the same time, the use of statistical methods in pharmacy and medicine has considerably developed to the point where it is today one of the main fields of application of experimental designs.

Recently, there has been considerable interest in the use of certain methods of local control called spatial or $m$ th order nearest-neighbor, abbreviated by ( $\mathrm{NN}$ or $\mathrm{NN} m$ ) where the integer $m>0$ is the distance among neighbours in the model. Kiefer and Wynn (1981) studied the optimality of block designs under a first order (NN1) neighbor correlation model, using the ordinary least squares (OLS) estimator. In this context, they have given sufficient conditions weak universally optimality for the estimation of treatment contrasts. Morgan and Chakravarti (1988) extend these conditions to the model NN2 and later Koné and Valibouze (2011) generalized this results to any correlation structure $\mathrm{NN} m, m>0$.

When the covariance structure is known, it is natural to construct the optimal experimental designs by making use of the generalized least squares (GLS) estimator. Among other authors, Kunert (1985, 1987), Azzalini and Giovagnoli (1987), Gill and Shukla (1985a b), Martin and Eccleston (1991), Grondona and Cressie (1993) and Satpati and al. (2007) have used this approach for the correlation structures $\operatorname{AR}(1)$ and $\operatorname{AR}(2)$, the autoregressive processes of first and second order (see Definition in Section 2.2). Kiefer and Wynn (1981) justified the choice of the OLS estimator, rather than the GLS estimator, in showing that the loss of accuracy relative resulting from the use of this estimator (OLS instead of the GLS) is quite low for the NN1 correlation models they consider.

The goal of this paper, is to find, for any integer $m \geq 1$, conditions of universal optimality block designs when errors correlation are modeled by an autoregressive $\operatorname{AR}(m)$ process for the estimation of treatment contrasts when generalized least squares estimation is used.

Section 2 presents the experimental context: the design and the correlation structure $\operatorname{AR}(m)$. Section 3 is devoted to the presentation of the model describing the correlation structure. Section 4 presents a known result about universally optimal designs due to Kiefer (1975a) and Section 5 contains the main theorem, the Optimality Theorem 5.1, which gives sufficient conditions for universal optimality. In Section 6, our Optimality Theorem is illustrated with the particular designs called semi balanced arrays. Section 7 is devoted to proofs.

\section{EXPERIMENTAL CONTEXT}

2.1. Designs for the considered experimental situation. We consider experimental situations in which $v \geq 1$ treatments are applied to $b \geq 1$ patients during $k \geq 1$ distinct 
periods. The generic term of period means the distinct unities of times where the treatments are applied. The following figure illustrates a such designs $d$ :

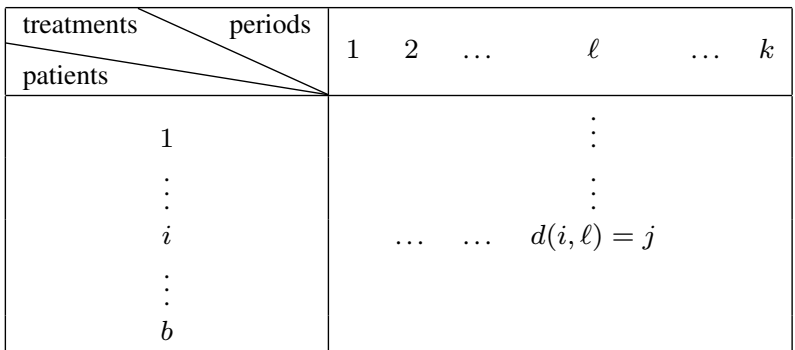

where $d(i, \ell)=j$ is one of the $v$ treatments applied to the $i$ th patient in the $\ell$ th period.

The goal is to construct the best experimental structure, the effectiveness being measured by optimality criteria evaluating the precision with which one considers the average value of the effects. The precision is measured by the information matrix of the estimates.

We denote by $\Omega_{v, b, k}$ the set of the block designs of this type. More precisely, we will reason within the framework of an experimental structure in incomplete blocks where a given patient, represented by a block, receives exactly $k$ distinct treatments distributed during the various periods. In each one of these periods, the patient receives only one of the $v$ treatments. This gives a single scalar experimental measurement.

Following the nomenclature of Morgan and Chakravarti (1988) and Grondona and Cressie (1993), we qualify by NNm-balanced the experimental designs in incomplete block, when they are balanced for distinct periods in time at most $m$ units (see lower exact Definition 1). In medical domain, the temporal balancing procedure can eliminate experimental results through the resulting effects interactions caused by the proximity in time of the administration of certain treatments on the same patient.

Definition 1. For an integer $m(m \geq 1)$ fixed, a design will by said $\mathrm{NN} m$-balanced (or a balanced design for nearest-neighbor at distance $m$ ) if for any integer $\delta \in \llbracket 1, m \rrbracket$, the number of times that two distinct treatments administered to the same patient are neighbors at distance $\delta$ is independent of the choice of these two treatments; that is to say, this value depends only on $\delta$ and on the design.

Example 2. The design of Table 1 bellow is NN2-balanced. Indeed, here $m=2$ and each pair of distinct treatments appears exactly three times at distance $\delta=1$ and 2 times at distance $\delta=2$. 
TABLE 1. NN2-balanced design

\begin{tabular}{|llll|}
\hline 1 & 2 & 3 & 4 \\
3 & 1 & 4 & 2 \\
1 & 4 & 3 & 2 \\
3 & 1 & 2 & 4 \\
1 & 2 & 4 & 3 \\
4 & 1 & 3 & 2 \\
\hline
\end{tabular}

2.2. Autoregressive correlation structure $\mathbf{A R}(m)$. Now we describe the correlation structure considered in this paper. We suppose that the correlation between observations carried out on distinct patients is null.

Let $\varepsilon=\left(\varepsilon_{1,1}, \ldots, \varepsilon_{1, k}, \ldots, \varepsilon_{i, 1}, \ldots, \varepsilon_{i, k}, \ldots, \varepsilon_{b, 1}, \ldots, \varepsilon_{b, k}\right)^{\prime}$ be the $b k$-vector of random errors where $\varepsilon_{i, \ell}$ is the process of error obtained at the $\ell$ th period $(\ell \in \llbracket 1, k \rrbracket)$ on the $i$ th patient $(i \in \llbracket 1, b \rrbracket)$ (see the model (6) that we consider). We suppose that $\varepsilon_{i, \ell}$ is a partial realization from a $m$ th order autoregressive process $\operatorname{AR}(m)$ characterized by the relations

$$
\varepsilon_{i, \ell}-\sum_{r=1}^{m} \theta_{r} \varepsilon_{i, \ell-r}=w_{i, \ell} \quad \text { for } \quad \ell=0, \pm 1, \pm 2, \ldots, \pm \infty
$$

where the $\theta_{r}$ are the parameters of the model and the $w_{i, \ell}$ are independently and identically distributed, null mean random variables with constant variance $\sigma^{2}$. The covariance function $\gamma$ of a process $\operatorname{AR}(m)$ satisfies the difference equation below (see, for example, Wei (1990)):

$$
\gamma(s)-\sum_{r=1}^{m} \theta_{r} \gamma(s-r)= \begin{cases}0 & \text { for } s>0 \\ \sigma^{2} & \text { for } s=0\end{cases}
$$

where for $i$ fixed in $\llbracket 1, b \rrbracket$ and for all $\ell \in \llbracket 1, k \rrbracket$

$$
\gamma(s)=\operatorname{Cov}\left(\varepsilon_{i, \ell}, \varepsilon_{i, \ell+s}\right) \text {. }
$$

In this paper, we will consider the variance-covariance matrix $V=\operatorname{Var}\left(\varepsilon_{i}\right)$ where $\varepsilon_{i}=$ $\left(\varepsilon_{i, 1}, \ldots, \varepsilon_{i, k}\right)^{\prime}$ is the error vector from the $i$ th patient. The matrix $V$ does not depend on the patient (it is the same for all the patients). The total variance-covariance matrix $V^{*}=\operatorname{Var}(\varepsilon)$ is given by:

$$
V^{*}=\mathbf{I}_{b} \otimes V
$$

where $\otimes$ is the Kronecker product and $\mathbf{I}_{b}$ is the $b \times b$ identity matrix.

\section{DESCRIPTION OF THE MODEL}

A design $d \in \Omega_{v, b, k}$ will be defined as a function

$$
d:(i, \ell) \in \llbracket 1, b \rrbracket \times \llbracket 1, k \rrbracket \rightarrow d(i, \ell) \in \llbracket 1, v \rrbracket
$$


where $d(i, \ell)$ is the treatment applied to the $i$ th $(1 \leq i \leq b)$ patient in the $\ell$ th $(1 \leq \ell \leq k)$ period. This design $d$ is presented by a $b \times k$ table of indices of the treatment taken among the $v$ possible values and applied to the different patients. In this table, the indices of the lines correspond to the $b$ patients, those columns to the $k$ periods of application and the treatment $d(i, \ell)$ is line $i$, column $\ell$.

We adopt the following notations: for a design $d, n_{d, j, i}$, is the number of times that the treatment $j$ is applied to the $i$ th patient, $r_{d, j}$ is the number of times the treatment $j$ is replicated in all of the experiment, $k_{d, i}$ is the total number of treatments received by the $i$ th patient and $\lambda_{d, j, j^{\prime}}$ is the number of patients for whom the distinct treatments $j$ and $j^{\prime}$ are both applied. For a fixed design $d$, the designation of $d$ in those notations would be omit.

If the design $d$ is such that $n_{d, j, i} \in\{0,1\}, r_{d, j}=r_{d, j^{\prime}}=r$ or $k_{d, i}=k_{d, i^{\prime}}=k$ it is respectively called binary, equireplicated and proper. For such designs the following identity holds

$$
r v=k b
$$

because each member of this equality counts the cardinality of the design $d$.

Throughout this paper, we restrict $\Omega_{v, b, k}$ to the set of binary, proper and equireplicated block designs. In such designs, each treatment is replicated exactly $r=\frac{k b}{v}$ times in the whole design and the $i$ th patient receives $k$ distinct treatments $d(i, 1), \ldots, d(i, k)$ successively applied in the periods $1, \ldots, k$. Moreover we have:

$$
\lambda_{d, j, j^{\prime}}=\sum_{i=1}^{b} n_{d, j, i} n_{d, j^{\prime}, i} .
$$

For optimality criteria we will consider the particular cases of BIBD and CBD defined as follows:

Definition 3. A binary proper equireplicated block design $d$ such that for each pair of distinct treatments $j, j^{\prime}$ the number $\lambda_{d, j, j^{\prime}}$ is a constant $\lambda$ is called a balanced incomplete block design, denoted by $\operatorname{BIBD}(v, b, r, k, \lambda)$. If moreover $k=v$ and $\lambda=r$, the design is called a complete block design, denoted by $\operatorname{CBD}(v, b)$.

The BIBD have been introduced and studied by Yates during the years 1936 to 1940 .

In our study, we consider the following classical linear model:

$$
\mathbf{Y}_{d}=\mu \mathbf{1}_{b k}+\left(\mathbf{I}_{b} \otimes \mathbf{1}_{k}\right) \alpha+\mathbf{T}_{d} \beta+\varepsilon
$$

where $\mathbf{1}_{a}$ and $\mathbf{1}_{a \times a}$ are respectively the $a$-vector and the $(a \times a)$-matrix composed entirely of one (for zero, we will adopt the same such notation with $\mathbf{0}_{a}$ ) 
- $\varepsilon$ is the $b k$-vector of random errors which follows the $\operatorname{AR}(m)$ process, zero mean: $\mathbb{E}(\varepsilon)=0$ and of variance-covariance matrix $\operatorname{Var}(\varepsilon)=V^{*}$ (see Section 2.2);

- in $\mathbf{Y}_{d}=\left(Y_{1,1}, \ldots, Y_{1, k}, \ldots, Y_{i, 1}, \ldots, Y_{i, \ell}, \ldots, Y_{i, k}, \ldots, Y_{b, 1}, \ldots, Y_{b, k}\right)^{\prime}$, the element $Y_{i, \ell}$ is the response of the $i$ th patient in the $\ell$ th period;

- $\mu$ represents the overall mean;

- $\alpha=\left(\alpha_{1}, \ldots, \alpha_{b}\right)^{\prime}$ is the vector of patient effects;

- $\beta$ is the $v$-vector of (uncorrected) treatment effects;

- the matrix $\mathbf{I}_{b} \otimes \mathbf{1}_{k}$ is interpreted as the $b k \times b$ incidence matrix;

- the $b k \times v$ incidence matrix $\mathbf{T}_{d}$ of periods-treatments is determined as follows:

(7) $\quad \mathbf{T}_{d}=\left[\begin{array}{c}T_{1} \\ \vdots \\ T_{b}\end{array}\right] \quad$ where $T_{i}=\left(\mathbf{t}_{\ell, j}(i)\right)_{1 \leq \ell \leq k, 1 \leq j \leq v} \quad$ and $\quad \mathbf{t}_{\ell, j}(i)=\delta_{j, d(i, \ell)}$

( $\delta_{a, b}$ is the Kronecker symbol).

Example 4. : For $v=k=3$ treatments and $b=2$ patients, we have:

$$
\left(\mathbf{I}_{b} \otimes \mathbf{1}_{k}\right)=\left(\begin{array}{cc}
1 & 0 \\
0 & 1 \\
1 & 0 \\
0 & 1 \\
1 & 0 \\
0 & 1
\end{array}\right) \text { and } T_{d}=\left(\begin{array}{ccc}
1 & 0 & 0 \\
1 & 0 & 0 \\
0 & 1 & 0 \\
0 & 1 & 0 \\
0 & 0 & 1 \\
0 & 0 & 1
\end{array}\right)
$$

when the design $d$ is:

\begin{tabular}{|c|ccc|}
\hline$j \backslash \ell$ & 1 & 2 & 3 \\
\hline$\imath$ & & & \\
\hline 1 & 1 & 1 & 2 \\
2 & 2 & 3 & 3 \\
\hline
\end{tabular}

Let $\mathbb{M}$ be the $(k \times k)$-matrix $\sigma^{2} V^{-1}$ whose general form was given by Wise $(1955)$ and Siddiqui (1958). Passi (1976) gave another formulation of their result. In the following, we give a similar formulation that will prove convenient to calculate the information matrix when estimating a set of treatment contrasts defined in Section 4.

Lemma 5. Let be an integer $m \geq 1$ and assume that $k>2 m$. Put $\theta_{0}=-1$ and $\theta_{u}=0$ for all $u>m$. Then the matrix $\mathbb{M}=\left(\gamma_{\ell, \ell^{\prime}}\right)_{1 \leq \ell, \ell^{\prime} \leq k}=\sigma^{2} V^{-1}$ is given by:

$$
\gamma_{\ell, \ell^{\prime}}=\sum_{u=0}^{\ell-1} \theta_{u} \theta_{u+\left(\ell^{\prime}-\ell\right)} \quad \text { for } \ell \in \llbracket 1, k-m \rrbracket \text { and } \ell^{\prime} \in \llbracket \ell, k \rrbracket
$$


More precisely and considering the zero values of the $\theta_{i}=0$, we have:

$\gamma_{\ell, \ell^{\prime}}: \begin{cases}(i) \gamma_{\ell, \ell}=\sum_{u=0}^{\ell-1} \theta_{u}^{2} & \text { for } \ell \in \llbracket 1, m \rrbracket \\ (i i) \gamma_{\ell, \ell}=\sum_{u=0}^{m} \theta_{u}^{2} & \text { for } \ell \in \llbracket m+1, k-m \rrbracket \\ (i i i) \gamma_{\ell, \ell+s}=\sum_{u=0}^{\ell-1} \theta_{u} \theta_{u+s} & \text { for } \ell \in \llbracket 1, m-1 \rrbracket \text { and } s \in \llbracket 1, m-\ell \rrbracket \\ (i v) \gamma_{\ell, \ell+s}=\sum_{u=0}^{m-s} \theta_{u} \theta_{u+s} & \text { for } \ell \in \llbracket 1, k-m \rrbracket \text { and } s \in \llbracket m a x(1, m-\ell+1), m \rrbracket \\ (v) \gamma_{\ell, \ell+s}=0 & \text { for } \ell \in \llbracket 1, k-m \rrbracket \text { and } s \in \llbracket m+1, k-\ell \rrbracket .\end{cases}$

The other elements not concerned by the formula (8) are determined by:

$$
\gamma_{\ell, \ell^{\prime}}=\gamma_{\ell^{\prime}, \ell}=\gamma_{k-\ell+1, k-\ell^{\prime}+1} \forall \ell, \ell^{\prime} \in \llbracket 1, k \rrbracket
$$

which means that the matrix is symmetrical with respect to its two diagonals.

Example 6. For $m=3$ and $k>6=2 m$, the matrix $\mathbb{M}$ of lemma 5 is given by

$\mathbb{M}=\left(\begin{array}{cccccccc}\theta_{0}^{2} & -\theta_{1} & -\theta_{2} & -\theta_{3} & 0 & \cdots & 0 & 0 \\ -\theta_{1} & \theta_{0}^{2}+\theta_{1}^{2} & -\theta_{1}+\theta_{1} \theta_{2} & -\theta_{2}+\theta_{1} \theta_{3} & -\theta_{3} & \cdots & 0 & 0 \\ -\theta_{2} & -\theta_{1}+\theta_{1} \theta_{2} & \theta_{0}^{2}+\theta_{1}^{2}+\theta_{2}^{2} & -\theta_{1}+\theta_{1} \theta_{2}+\theta_{2} \theta_{3} & -\theta_{2}+\theta_{1} \theta_{3} & \cdots & 0 & 0 \\ -\theta_{3} & -\theta_{2}+\theta_{1} \theta_{3} & -\theta_{1}+\theta_{1} \theta_{2}+\theta_{2} \theta_{3} & \theta_{0}^{2}+\theta_{1}^{2}+\theta_{2}^{2}+\theta_{3}^{2} & -\theta_{1}+\theta_{1} \theta_{2}+\theta_{2} \theta_{3} & \cdots & 0 & 0 \\ \vdots & & & & & & & \vdots \\ 0 & 0 & 0 & 0 & 0 & \cdots & \theta_{0}^{2}+\theta_{1}^{2} & -\theta_{1} \\ 0 & 0 & 0 & 0 & 0 & \cdots & -\theta_{1} & -\theta_{0}\end{array}\right)$.

Now we present an essential lemma about the sum of elements of each line of the matrix M:

Lemma 7. Suppose $k>2 m$ and let $\ell \in \llbracket 1, k \rrbracket$. Put $p_{\ell}=\sum_{\ell^{\prime}=1}^{k} \gamma_{\ell, \ell^{\prime}}$, the sum of the elements of the line $\ell$ of the matrix $\mathbb{M}, a_{\ell}=\sum_{u=\ell}^{m} \theta_{u}$ for $\ell \leq m$ and $a_{\ell}=0$ for $\ell>m$. Then: 


$$
p_{\ell}=a_{0}\left(a_{0}-a_{\ell}\right)=\left(1-\theta_{1}-\cdots-\theta_{m}\right)\left(1-\theta_{1}-\cdots-\theta_{\ell-1}\right)
$$

for $\ell \in \llbracket 1, k-m \rrbracket$ and, as $\mathbb{M}$ is symmetric with respect to its second diagonal, $p_{\ell}=$ $p_{k-\ell+1}$ for $\ell \in \llbracket k-m, k \rrbracket$. In particular, $p_{\ell}=p_{m+1}=a_{0}^{2}$ for $\ell \in \llbracket m+1, k-m \rrbracket$.

Recall that Section 7 is devoted to the proofs.

The expression of $p_{\ell}$ given in Lemma 7 will be introduce in the proof of expressions of diagonal and extra-diagonal elements of the information matrix $C_{d}$ that are given in Lemma 12, moreover, in this lemma, the coefficient $c$ appearing in the expressions is the sum of all elements of the matrix $\mathbb{M}$ given by:

$$
c=\mathbf{1}^{\prime} \mathbf{M} \mathbf{1}=2 a_{0} \sum_{\ell=0}^{m-1}(m-\ell) \theta_{\ell}+(k-2 m) a_{0}^{2}
$$

because

$$
\begin{aligned}
c & =\sum_{\ell=1}^{k} \sum_{\ell^{\prime}=1}^{k} \gamma_{\ell, \ell^{\prime}}=2 \sum_{\ell=1}^{m} p_{\ell}+\sum_{\ell=m+1}^{k-m} p_{\ell}=2 a_{0} \sum_{\ell=1}^{m}\left(a_{0}-a_{\ell}\right)+\sum_{\ell=m+1}^{k-m} p_{m} \\
& =2 a_{0} \sum_{\ell=1}^{m}\left(\theta_{0}+\theta_{1}+\cdots+\theta_{\ell-1}\right)+(k-2 m) a_{0}^{2}=2 a_{0} \sum_{\ell=0}^{m-1}(m-\ell) \theta_{\ell}+(k-2 m) a_{0}^{2} .
\end{aligned}
$$

\section{ESTIMATION OF THE TREATMENT EFFECTS}

In this section, we give the information matrix of a set of contrasts of effects related to the treatments.

Consider the $i$ th patient, $i \in \llbracket 1, b \rrbracket$, and put $Y_{i}=\left(Y_{i, 1}, \ldots, Y_{i, \ell}, \ldots, Y_{i, k}\right)^{\prime}$, the vector of the $k$ responses of the $i$ th patient. In the model $(6)$, the GLS estimator $\widehat{\beta}$ of the $v$-vector $\beta$ of treatment effects satisfies the following reduced normal equations (see Benchekroun (1993)):

$$
T_{i}^{\prime} \mathbb{W} T_{i} \beta=T_{i}^{\prime} \mathbb{W} Y_{i} \quad \text { where } \quad \mathbb{W}=V^{-1}-\left(\mathbf{1}_{k}^{\prime} V^{-1} \mathbf{1}_{k}\right)^{-1} V^{-1} \mathbf{1}_{k} \mathbf{1}_{k}^{\prime} V^{-1}
$$

As said before Lemma 5 concerning the matrix $\mathbb{M}$, we are interested in the estimation of a set of treatment contrasts. Let $\gamma \in \mathbb{R}^{v}$. A linear combination $\gamma^{\prime} \cdot \beta=\sum_{j=1}^{v} \gamma_{j} \beta_{j}$, where $\beta$ is a treatment effects, is called a treatment contrast if $\gamma^{\prime} \cdot \mathbf{1}_{v}=0$. A block design $d$ is said to connected if all treatment contrasts are estimable under $d$. We refer to Dey (2010) (pages 11 and 12) for the details of connected designs. 
In the following, we restrict $\Omega_{v, b, k}$ at block designs which are binary, equireplicate, proper and connected.

As, the vector $\gamma=\left(\beta_{j}-\frac{1}{v} \sum_{j=1}^{v} \beta_{j}\right)_{1 \leq j \leq v}$ of corrected treatment effects satisfies the identifiability constraint $\gamma^{\prime} \cdot \mathbf{1}_{v}=0$, the optimality results of a design $d \in \Omega_{v, b, k}$ will be based on the set of contrasts

$$
\gamma=\left(\mathbf{I}_{v}-\frac{\mathbf{1}_{v \times v}}{v}\right) \beta
$$

Observe that a such choice is suitable since $u^{\prime} \cdot \gamma=u^{\prime} \cdot \beta$ for any $u \in \mathbb{R}^{v}$ such that $u^{\prime} \cdot \mathbf{1}_{v}=0$. Denote by $A^{\dagger}$ the Moore-Penrose inverse of a matrix $A$ and by $\widehat{\gamma}$ the GLS estimator of a contrast $\gamma$. Then from (12) and (13) (see Grondona and Cressie (1993)):

$$
\widehat{\gamma}=\left(\mathbf{I}_{v}-\frac{\mathbf{1}_{v \times v}}{v}\right)\left(T_{i}^{\prime} \mathbb{W} T_{i}\right)^{\dagger} T_{i}^{\prime} \mathbb{W} Y_{i}=\left(T_{i}^{\prime} \mathbb{W} T_{i}\right)^{\dagger} T_{i}^{\prime} Y_{i}
$$

From equation (14), for the $i$ th patient, the variance-covariance matrix of $\widehat{\gamma}$ is given by: $\operatorname{Var}(\widehat{\gamma})=\left(T_{i}^{\prime} \mathbb{W} T_{i}\right)^{\dagger}$. Then, for the $i$ th patient, the information matrix $\mathbf{C}_{i, d}(V)=\operatorname{Var}(\widehat{\gamma})^{\dagger}$ of $\widehat{\gamma}$ satisfies:

$$
\mathbf{C}_{i, d}(V)=T_{i}^{\prime} \mathbb{W} T_{i}
$$

It follows this lemma stated by Kunert (1987) (see detailed proof in Benchekroun (1993)):

Lemma 8. In the covariance structure (1,2, 3, the information matrix of the estimator $\widehat{\gamma}$ of treatment contrasts is given by

$$
\boldsymbol{C}_{d}\left(V^{*}\right)=\sum_{i=1}^{b} T_{i}^{\prime} \mathbb{W} T_{i}
$$

where $V^{*}=\mathbf{I}_{b} \otimes V$ and $\mathbb{W}=\left(w_{\ell, \ell^{\prime}}\right)_{1 \leq \ell, \ell^{\prime} \leq k}=V^{-1}-\left(\mathbf{1}_{k}^{\prime} V^{-1} \mathbf{1}_{k}\right)^{-1} V^{-1} \mathbf{1}_{k} \mathbf{1}_{k}^{\prime} V^{-1}$.

\section{UNIVERSAL OPTIMALITY}

The study of the information matrix associated with the estimator $\widehat{\gamma}$ of treatment contrasts can build strong optimality criteria.

\subsection{Preliminary.}

Let $\mathcal{V}=\left\{\mathbf{C}_{d}: d \in \Omega_{v, b, k}\right\}$ be the set of information matrices for the estimators $\widehat{\gamma}$ of treatment contrasts associated with the design in question. For each design $d$, the information matrix $\mathbf{C}_{d}$ is positive semi-definite of dimension $v \times v$ and satisfies Identities (see Kiefer (1975b): 


$$
\mathbf{C}_{d} \mathbf{1}_{v}=\mathbf{C}_{d}^{\prime} \mathbf{1}_{v}=\mathbf{0}_{v}
$$

which says that $\mathbf{C}_{d}$ has row- and column-zero for all $d \in \Omega_{v, b, k}$.

Definition 9. (Kiefer (1975a)) A block design $d$ belonging to $\Omega_{v, b, k}$ is said universally optimal if its information matrix $C_{d}$ minimizes simultaneously all fonctions $\psi: \mathcal{V} \mapsto$ ] $-\infty,+\infty$ ], called criterions, satisfying the three following conditions:

(i) for each $\mathbf{C} \in \mathcal{V}, \psi(\mathbf{C})$ is invariant under all permutations applied to the rows and columns of $\mathbf{C}$;

(ii) $\psi$ is convex, i.e. $\psi\left\{a \mathbf{C}_{1}+(1-a) \mathbf{C}_{2}\right\} \leq a \psi\left(\mathbf{C}_{1}\right)+(1-a) \psi\left(\mathbf{C}_{2}\right)$ for all $\mathbf{C}_{1}, \mathbf{C}_{2} \in \mathcal{V}$ and $0 \leq a \leq 1$

(iii) $\psi(a \mathbf{C}) \geq \psi(\mathbf{C})$ for all $\mathbf{C} \in \mathcal{V}$ once $0<a<1$.

Proposition 10. $($ Kiefer $(1975 b))$ Suppose that there exists $d^{*} \in \Omega_{v, b, k}$, such that its information matrix $\boldsymbol{C}_{d^{*}}$ has row-and column-zero and verifies: (i) $\boldsymbol{C}_{d^{*}}$ is completely symmetric, i.e. $\boldsymbol{C}_{d^{*}}=\alpha \boldsymbol{I}_{v}-\alpha / v \boldsymbol{I}_{v \times v}$ where $\alpha$ is a scalar and (ii) the trace of $\boldsymbol{C}_{d^{*}}$ is maximum on the set $\mathcal{V}$. Then the design $d^{*}$ is universally optimal in $\Omega_{v, b, k}$.

By Identity (16), we just have to find a design whose information matrix meets conditions (i) and (ii) of Kiefer's Theorem 10. We will seek them among the optimal designs.

\subsection{Optimality conditions for the correlation structure $\mathbf{A R}(m)$.}

Let $d$ be a block design in $\Omega_{v, b, k}$ and the integer $m>0$ such that $k>2 m$. We adopt the following notation which generalize those of Gill and Shukla (1985a) and Grondona and Cressie (1993) for the respective cases $m=1$ and $m=2$ :

$\phi_{d, j, i}^{\ell}$ is the number of times that the treatment $j$ is applied to the patient $i$ at the period $\ell$ or $(k-\ell+1)$ with $\ell \in \llbracket 1, m \rrbracket$;

$\phi_{d, j}^{\ell}$ is the number of patients for which the treatment $j$ is applied to the $\ell$ th or to the ( $k-$ $\ell+1)$ th period when $\ell \in \llbracket 1, m \rrbracket$ and to the $\ell$ th period when $\ell \in \llbracket m+1, k-m \rrbracket$;

$\phi_{d, j, j^{\prime}}^{\ell *}$ is the number of times that the treatments $j$ and $j^{\prime}$ occur together and $j$ or $j^{\prime}$ are applied to the $\ell$ th period or the $(k-\ell+1)$ th period for $\ell \in \llbracket 1, m \rrbracket$; (counted twice if $j$ and $j^{\prime}$ are applied to both these periods);

$N_{d, j, j^{\prime}, i}^{s}$ is the number of times that the treatments $j$ and $j^{\prime}$ are applied to the patient $i$ with a distance $s \in \llbracket 1, k-1 \rrbracket$; with $N_{d, j, j, i}^{s}=0$;

$N_{d, j, j^{\prime}}^{s}$ is the number of patients in which treatments $j$ and $j^{\prime}$ occur as $s$ th nearest neighbours (i.e. separate by $s-1$ period); with $N_{d, j, j}^{s}=0$.

Remark 11. When the design $d$ is binary, we have: $\phi_{d, j, i}^{\ell}=\delta_{j, d(i, \ell)}+\delta_{j, d(i, k-\ell+1)}$ and 


$$
N_{d, j, j^{\prime}, i}^{s}= \begin{cases}1 & \text { if } j \text { and } j^{\prime} \text { are applied with a distance } s \text { to the } i \text { th patient } \\ 0 & \text { otherwise }\end{cases}
$$

When the design $d$ is equireplicated, for each treatment $j \in \llbracket 1, v \rrbracket$, we have:

$$
r_{d, j}=\sum_{\ell=1}^{k-m} \phi_{d, j}^{\ell}=r
$$

In Example 23, some of this previous quantities are counted in a concrete design.

The following lemma generalizes to any $m$ the results (4.8) and (4.9) of Grondona and Cressie (1993) for $m=2$ :

Lemma 12. Let be a design $d \in \Omega_{v, b, k}$, for an integer $k$ which verifies $k>2 m$. Then, for the AR(m) model, the diagonal entries of the matrix $\boldsymbol{C}_{d}$ is given as follows for each treatment $j \in \llbracket 1, v \rrbracket$ :

$$
\sigma^{2} \boldsymbol{C}_{d, j, j}\left(V^{*}\right)=s_{0} r-s_{1} \phi_{d, j}^{1}-s_{2} \phi_{d, j}^{2}-\cdots-s_{m} \phi_{d, j}^{m}
$$

and taking $j^{\prime} \neq j$ in $\llbracket 1, v \rrbracket$, the off-diagonal entries are given by:

$$
\begin{aligned}
\sigma^{2} \boldsymbol{C}_{d, j, j^{\prime}}\left(V^{*}\right)= & \sum_{s=1}^{m} N_{d, j, j^{\prime}}^{s} \sum_{u=0}^{m-s} \theta_{u} \theta_{u+s} \\
& -\sum_{s=1}^{m-1} \sum_{t=1}^{m-s} \sum_{u=t}^{m-s} \theta_{u} \theta_{u+s} \sum_{i=1}^{b} N_{d, j, j^{\prime}, i}^{s}\left(\phi_{d, j, i}^{t} \phi_{d, j^{\prime}, i}^{t+s}+\phi_{d, j^{\prime}, i}^{t} \phi_{d, j, i}^{t+s}\right) \\
& -c^{-1} a_{0}^{2}\left\{a_{0}^{2} \lambda_{d, j, j^{\prime}}-a_{0} \sum_{\ell=1}^{m} a_{\ell} \phi_{d, j, j^{\prime}}^{\ell *}+\sum_{\ell=1}^{m} \sum_{\ell^{\prime}=1}^{m} a_{\ell} a_{\ell^{\prime}} \sum_{i=1}^{b} \phi_{j, i}^{\ell} \phi_{j^{\prime}, i}^{\ell^{\prime}}\right\}
\end{aligned}
$$

where

$$
\begin{aligned}
a_{\ell} & =\sum_{u=\ell}^{m} \theta_{u}, \quad b_{\ell}=\sum_{u=\ell}^{m} \theta_{u}^{2}, \\
c & =\boldsymbol{I}^{\prime} \mathbf{M} \boldsymbol{I}=2 a_{0} \sum_{\ell=0}^{m-1}(m-\ell) \theta_{\ell}+(k-2 m) a_{0}^{2} \quad \text { and } \\
s_{\ell} & =b_{\ell}+c^{-1} a_{0}^{2} a_{\ell}\left(a_{\ell}-2 a_{0}\right) ; \text { in particular, } s_{0}=b_{0}-c^{-1} a_{0}^{4} .
\end{aligned}
$$

\section{Remark 13. Consequence of lemma 12}


The trace $\operatorname{tr}\left(\mathbf{C}_{d}\right)=\sum_{j=1}^{v} \mathbf{C}_{d, j, j}(V)$ of the information matrix $\mathbf{C}_{d}$ of $\widehat{\gamma}$ is independent of choice of the design in $d \in \Omega_{v, b, k}$ and is given by the identity

$$
\sigma^{2} \operatorname{tr}\left(\mathbf{C}_{d}\right)=s_{0} v r-2 b\left(s_{1}+s_{2}+\cdots+s_{m}\right) .
$$

Indeed, following Lemma 12 and identity (21), we have:

$$
\begin{aligned}
\sigma^{2} \operatorname{tr}\left(\mathbf{C}_{d}\right) & =s_{0} v r-s_{1} \sum_{j=1}^{v} \phi_{d, j}^{1}-s_{2} \sum_{j=1}^{v} \phi_{d, j}^{2}-\cdots-s_{m} \sum_{j=1}^{v} \phi_{d, j}^{m} \\
& =s_{0} v r-2 b\left(s_{1}+s_{2}+\cdots+s_{m}\right)
\end{aligned}
$$

since we have this identity (Koné and Valibouze, 2011):

$$
\sum_{j=1}^{v} \phi_{d, j}^{\ell}=2 b \text { for } k>2 m \text { and } \ell \in \llbracket 1, m \rrbracket .
$$

So all the designs which are potentially optimal in $\Omega_{v, b, k}$ have the same trace. It remains to identify the designs of $\Omega_{v, b, k}$ with an information matrix which is completely symmetric, i.e., the designs which are universally optimal in $\Omega_{v, b, k}$.

Before the main Theorem, we establish three lemmas concerning terms of expression of the terms $\mathbf{C}_{d, j, j^{\prime}}$ given in Lemma 12. The first one in follows concerns the BIBD designs.

Lemma 14. Assume $k \leq v$. For a $B I B D(v, b, r, k, \lambda)$ design $d$, the number of times that two distinct treatments $j, j^{\prime}$ are administered to the same patient is a constant $\lambda$ given by

$$
\text { (i) } \quad \lambda=\lambda_{d, j, j^{\prime}}=\frac{b k(k-1)}{v(v-1)} \quad \forall j, j^{\prime} \in \llbracket 1, v \rrbracket, j \neq j^{\prime} .
$$

The second lemma concerns the $\mathrm{NN} m$-balanced designs:

Lemma 15. For a design $d N N m$-balanced and an integer $s \in \llbracket 1, m \rrbracket$, there exists a constant $N_{d}^{s}$ counting the number of times that two distinct treatments are applied to a same patient at a distance $s$ and this constant is given by:

$$
\text { (ii) } \quad N_{d}^{s}=N_{d, j, j^{\prime}}^{s}=\frac{2 b(k-s)}{v(v-1)} \quad \forall j, j^{\prime} \in \llbracket 1, v \rrbracket, j \neq j^{\prime}
$$

The third lemma concerning the square $\mathrm{NN} m$-balanced designs will be use for establishing Theorem 5.2.

Lemma 16. Assume that the designs are square, i.e. the number $k$ of periods equals the number $v$ of treatments. Then a design $d$ which is NNm-balanced satisfies not only identity 
(ii) of Lemma 15 but also the following:

$$
\begin{aligned}
\phi_{d, j, j^{\prime}}^{\ell *} & =\frac{4 b}{v} \quad \text { and } \\
\phi_{d, j}^{\ell} & =\frac{2 b}{v}
\end{aligned}
$$

for each $j, j^{\prime} \in \llbracket 1, v \rrbracket\left(j \neq j^{\prime}\right)$ and for each $\ell \in \llbracket 1, m \rrbracket$.

We can now establish our main Theorem:

Theorem 5.1 (Optimality Theorem). Assume $2 m<k<v$. If a NNm-balanced $\operatorname{BIBD}(v, b, r, k, \lambda) d \in \Omega_{v, b, k}$ for the model AR(m) exists, which also fulfills the conditions $(i i i),(i v)$ and $(v)$ below, for all distinct treatments $j$ and $j^{\prime}$ in $\llbracket 1, v \rrbracket$ and for $\ell, \ell^{\prime} \in \llbracket 1, m \rrbracket$, then $d$ is universally optimal over $\Omega_{v, b, k}$;

$$
\begin{aligned}
\phi_{d, j, j^{\prime}}^{\ell *} & =\frac{4 b(k-1)}{v(v-1)}, \\
\sum_{i=1}^{b} \phi_{d, j, i}^{\ell} \phi_{d, j^{\prime}, i}^{\ell^{\prime}} & =\frac{2 b\left(2-\delta_{\ell, \ell^{\prime}}\right)}{v(v-1)}
\end{aligned}
$$

$$
\sum_{i=1}^{b} N_{d, j, j^{\prime}, i}^{\ell \ell-\ell^{\prime} \mid}\left(\phi_{d, j, i}^{\ell} \phi_{d, j^{\prime}, i}^{\ell^{\prime}}+\phi_{d, j^{\prime}, i}^{\ell} \phi_{d, j, i}^{\ell^{\prime}}\right)=\frac{4 b}{v(v-1)} \quad \text { when } \ell \neq \ell^{\prime}
$$

where $\delta_{\ell, \ell^{\prime}}$ is the Kronecker symbol.

When $d$ is an $\mathrm{NN} m$-balanced universally optimal $\operatorname{BIBD}(v, b, r, k, \lambda)$, Optimality Theorem implies that the off-diagonal entries of the matrix $\mathbf{C}_{d}$ are given by:

$$
\begin{aligned}
\sigma^{2} \mathbf{C}_{d, j, j^{\prime}}\left(V^{*}\right)= & \frac{2 b(k-s)}{v(v-1)} \sum_{s=1}^{m} \sum_{u=0}^{m-s} \theta_{u} \theta_{u+s}-\frac{4 b}{v(v-1)} \sum_{s=1}^{m-1} \sum_{t=1}^{m-s} \sum_{u=t}^{m-s} \theta_{u} \theta_{u+s} \\
\text { (24) } & -\frac{b c^{-1} a_{0}^{2}}{v(v-1)}\left\{a_{0}^{2} k(k-1)-a_{0} 4(k-1) \sum_{\ell=1}^{m} a_{\ell}+2 \sum_{\ell=1}^{m}\left(a_{\ell}^{2}+2 a_{\ell} \sum_{\substack{\ell^{\prime}=1 \\
\ell^{\prime} \neq \ell}}^{m} a_{\ell^{\prime}}\right)\right\}
\end{aligned}
$$

where $j^{\prime} \neq j$ in $\llbracket 1, v \rrbracket$,

$$
a_{\ell}=\sum_{u=\ell}^{m} \theta_{u} \quad \text { and } \quad c=\mathbf{1}^{\prime} \mathbb{M} \mathbf{1}=2 a_{0} \sum_{\ell=0}^{m-1}(m-\ell) \theta_{\ell}+(k-2 m) a_{0}^{2}
$$

In the case of a complete block design, we obtain the optimality following result:

Theorem 5.2. Suppose $k>2 m$ and the designs are square: the number $k$ of periods equals the number $v$ of treatments. If a NNm-balanced $C B D(v, b) d \in \Omega_{v, b, k}$ for the $A R(m)$ 
model exists then $d$ is universaly optimal over $\Omega_{v, b, k}$ if it fulfills the conditions $(i v)$ and $(v)$ of Theorem 5.1 .

Remark 17. A necessary condition for the existence of the designs satisfying the condition conditions $(i i i)-(v)$ of Theorem 5.1 and Theorem 5.2 is then $v(v-1) \mid 2 b$. A minimal design corresponds to the minimal value of $b$ for $k$ and $v$ fixed satisfying the associated optimality conditions. Morgan and Chakravarti (1988) and Koné and Valibouze (2011) showed that the minimal value of $b$ for wich the existe a NN2-balanced CBD is $b=$ $v(v-1) / 2$.

In Lemma 12, Theorem 5.1 and Theorem 5.2, for the value $m=1$ we find the results of Kunert (1987) and for value $m=2$ we find the results of Grondona and Cressie (1993) 1

In the section below, the Optimality Theorem is illustrated with well-known designs called semi balanced arrays.

\section{Optimality Theorem And SEmi BALANCED ARRAYS}

Rao (1946, 1947) introduced the concept of orthogonal arrays denoted by OA. Two variants of them are defined by Rao (1961): the OA of Type I and the OA of Type II which are renamed respectively transitive arrays, denoted by TA, and semi balanced arrays, denoted by SB. For more details, see Rao (1961, 1973), Morgan and Chakravarti (1988) and Hedayat, Sloane et Stufken (1999).

Definition 18. A $b \times k$ array of $v$ symbols is an $\mathrm{SB}(b, k, v, t)$, where $t$ is called its strength, if in every set of $t$ columns each unordered $t$-tuples have distinct symbols among $v$ and appears exactly $\omega$ times, where $\omega \in \mathbb{N}$ is called the index of the SB.

To define a TA, it suffices to replace "unordered $t$-tuples" by "ordered $t$-tuples" in the definition of a SB. Clearly, a TA $(b, k, v, t)$ of index $\omega$ is an $\operatorname{SB}(b, k, v, t)$ of index $t$ ! $\omega$.

Remark 19. As the number of unordered $t$-tuples of symbols is $\left(\begin{array}{l}v \\ t\end{array}\right)=\frac{v !}{t !(v-t) !}$, the index of an $\operatorname{SB}(b, k, v, t)$ verifies $\omega=b /\left(\begin{array}{l}v \\ t\end{array}\right)$. By consequence, the existence of an $\operatorname{SB}(b, k, v, t)$ implies that $b$ is the multiple $\omega\left(\begin{array}{l}v \\ t\end{array}\right)$ of $\left(\begin{array}{l}v \\ t\end{array}\right)$.

Note that in the context of our paper, $b$ is the number of patients, $k$ is the number of treatments received by patient, and the $v$ symbols represent the indices of the distinct treatments. We have chosen this context to simplify our explanations.

\footnotetext{
${ }^{1}$ without one error of their paper in one element of the summation (4.9) giving $\sigma^{2} c_{l, m}$ with $l \neq m$ : according to their notations the factor 2 in $2\left(1-\phi_{1}-\phi_{2}\right) \phi_{2} f_{l, m}^{*}$ must be removed and, moreover, their definitions of $f_{l, m}^{*}$ and $e_{l, m}^{*}$ must be modified as follows: $e_{l, m}^{*}:=\phi_{d, l, m}^{1 *}$ and $f_{l, m}^{*}:=\phi_{d, l, m}^{2 *}$.
} 
Many methods for constructing SB and TA exist in the literature (Morgan and Chakravarti (1988), Mukhopadhyay (1972), Ramanujacharyulu (1966), Stufken (1991), ...). In context of this section, we will be interested only in the strength $t=2$. Rao (1961, 1973) showed that if a TA $(v(v-1), k, v, 2)$ exists, then it can be constructed from $(k-1) \mathrm{mu}$ tually orthogonal Latin squares of order $v$, and that if $v$ is an odd prime power or an odd prime, an $\operatorname{SB}(v(v-1) / 2, v, v, 2)$ can be constructed from $\operatorname{GF}(v)$, the Galois field with $v$ elements.

Example 20. Below, the designs have been constructed by Deheuvels and Derzko (1991).

\begin{tabular}{|c|c|c|c|c|c|c|c|c|c|c|c|c|c|c|c|c|c|c|c|}
\hline 1 & 3 & 4 & 5 & 2 & 1 & 4 & 3 & 2 & & & & & & & & & & & \\
\hline 2 & 4 & 5 & 1 & 3 & 2 & 5 & 4 & 3 & 1 & & & & & & & & & & \\
\hline 3 & 5 & 1 & 2 & 4 & 3 & 1 & 5 & 4 & 2 & & & & & & & & & & \\
\hline 4 & 1 & 2 & 3 & 5 & 4 & 2 & 1 & 5 & 3 & 1 & 3 & 4 & 5 & & & 2 & 5 & 3 & \\
\hline 5 & 2 & 3 & 4 & 1 & 5 & 3 & 2 & 1 & 4 & 2 & 4 & 5 & 1 & 3 & 2 & 3 & 1 & 4 & 5 \\
\hline 1 & 2 & 5 & 3 & 4 & 1 & 5 & 2 & 4 & 3 & 3 & 5 & 1 & 2 & 4 & 3 & 4 & 2 & 5 & 1 \\
\hline 2 & 3 & 1 & 4 & 5 & 2 & 1 & 3 & 5 & 4 & 4 & 1 & 2 & 3 & 5 & 4 & 5 & 3 & 1 & 2 \\
\hline 3 & 4 & 2 & 5 & 1 & 3 & 2 & 4 & 1 & 5 & 5 & 2 & 3 & 4 & 1 & 5 & 1 & 4 & 2 & 3 \\
\hline 4 & 5 & 3 & 1 & 2 & 4 & 3 & 5 & 2 & 1 & \multirow{2}{*}{\multicolumn{10}{|c|}{ (c) }} \\
\hline 5 & 1 & 4 & 2 & 3 & 5 & 4 & 1 & 3 & 2 & & & & & & & & & & \\
\hline
\end{tabular}

The design (a), obtained by superposition of the two quasi-complete Latin squares (c) and (d), is an $\mathrm{SB}(10,5,5,2)$ of index 1 . The design (b) is superposition of mirror image of designs (c) and (d). The design (e) resulting to the superposition of designs (a) and (b) yields a TA $(20,5,5,2)$ of index 1 or a $\mathrm{SB}(20,5,5,2)$ of index 2 .

Martin and Eccleston (1991) have introduced SDEN designs (SDEN for strongly directionally equineighboured $)^{2}$. They remark that for $k \geq 3$ a $\operatorname{SDEN}(b, k, v)$ is equivalent to a $\mathrm{SB}(b, k, v, 2)$ and construct these designs from $\omega(v-1)$ latin squares of order $v$. The part (a) of they Theorem 2 implies that for $k \geq 3$ if an $\mathrm{SB}(b, k, v, 2)$ exists then it is universally optimal within $\Omega_{v, b, k}$ for any variance-covariance matrix $V$, under GLS.

We have interested to SB's of strength $t=2$ since if an $\mathrm{SB}(b, k, v, 2)$ exists then it can be interpreted as a $\operatorname{BIBD}(v, b, r, k, \lambda)$ (it is well kwown and easily provable). The following proposition applies Theorem 2 in Martin and Eccleston (1991). These authors use a broader correlation model than $\operatorname{AR}(m)$, but that applies only (for optimality) to a subset of designs among those we study in this article. Not that this theorem also applied to non-binary designs that we do not consider.

\footnotetext{
${ }^{2}$ Deheuvels and Derzko (1991) used the terms totally balanced for SDEN and SB, and universally balanced for TA.
} 
Proposition 21. Suppose that there exists an $S B(b, k, v, 2)$ and call it $d$. Then $d$ is a $\operatorname{BIBD}(v, b, r, k, \lambda)$ with

(25) $\lambda=\frac{k(k-1)}{2} \omega \quad$ and $\quad r=\frac{k(v-1)}{2} \omega \quad$ where $\quad \omega=b \frac{2}{v(v-1)}$

is the index of $d$ and it is NNm-balanced universally optimal for the AR $(m)$ correlation structure. In particular, for $j, j^{\prime} \in \llbracket 1, v \rrbracket, j \neq j^{\prime}$ and $\ell, \ell^{\prime}, s \in \llbracket 1, m \rrbracket$, $d$ verifies:

$$
\begin{aligned}
N_{d}^{s}=N_{d, j, j^{\prime}}^{s} & =\omega(k-s) \\
\phi_{d, j, j^{\prime}}^{\ell *} & =2 \omega(k-1) \\
\sum_{i=1}^{b} \phi_{d, j, i}^{\ell} \phi_{d, j^{\prime}, i}^{\ell^{\prime}} & =\omega\left(2-\delta_{\ell, \ell^{\prime}}\right) \\
\sum_{i=1}^{b} N_{d, j, j^{\prime}, i}^{\left|\ell-\ell^{\prime}\right|}\left(\phi_{d, j, i}^{\ell} \phi_{d, j^{\prime}, i}^{\ell^{\prime}}+\phi_{d, j^{\prime}, i}^{\ell} \phi_{d, j, i}^{\ell^{\prime}}\right) & =2 \omega \quad \text { when } \ell \neq \ell^{\prime} .
\end{aligned}
$$

Morgan and Chakravarti (1988) (Theorems 4.1) and Koné and Valibouze (2011) gave a similar result in the case of the weakly universally optimal BIBD for respectively the NN2 and $\mathrm{NN} m$ (for all $m>1$ ) correlation structure.

Remark 22. Let $j, j^{\prime} \in \llbracket 1, v \rrbracket, j \neq j^{\prime}$ and $m<2 k$. Recall that a $\operatorname{BIBD}(v, b, k, \lambda)$ is $\mathrm{NN} m$-balanced for the $\operatorname{AR}(m)$ model if the quantity $N_{d, j, j^{\prime}}^{s}$ is independent to $j$ and $j^{\prime}$ (see Lemma 15 and it is universally optimal if moreover the three quantities at left of equalities (iii), $(i v)$ and $(v)$ of Optimality Theorem 5.1 are independent to $j$ and $j^{\prime}$ and it this case the three equalities hold.

Proof. As it is known that an $\operatorname{SB}(b, k, v, 2)$ can be interpreted as a $\operatorname{BIBD}(v, b, r, k, \lambda)$, the identities in (25) come from Remark (19) and Identities $r v=b k$ in (4) and $\lambda(v-1)=$ $r(k-1)$ in Lemma 14 . Now consider an unordered pair $\left(j, j^{\prime}\right)$ of two distinct treatments.

For all $m \in \llbracket 1, k-1 \rrbracket$, the design $d$ is $\mathrm{NN} m$-balanced because $N_{d, j, j^{\prime}}^{s}$, the number of times that $\left(j, j^{\prime}\right)$ are applied to a same patient at distance $s \in \llbracket 1, m \rrbracket$, is a constant $N_{d}^{s}$. More precisely, considering the $k-s$ pairs of periods $\ell$ and $\ell+s$ where $\ell$ runs in $\llbracket 1, k-s \rrbracket$, as the strength of $d$ is two, we obtain Identity (27): $N_{d}^{s}=N_{d, j, j^{\prime}}^{s}=\omega(k-s)$. As $2 b=\omega v(v-1)$, $N_{d}^{s}$ is obviously the same constant as that given in Lemma 15 .

To prove the rest of Proposition 21, we apply (a) of Theorem 2 in Martin and Eccleston (1991) which implies that $d$ is universally optimal and replace $2 b$ by $\omega v(v-1)$ in Identities (iii), (iv) and (v) of Optimality Theorem 5.1 (see Remark 22). 
We illustrate both our Optimality Theorem 5.1 and Proposition 21 by the following example.

Example 23. Verify identities of Proposition 21 on design (a) of Example 20. This design is an $\operatorname{SB}(b=10, k=5, v=5, t=2)$ of index $\omega=1$. We consider (a) as a $\operatorname{BIBD}(v=5, b=10, r=10, k=5, \lambda=10)$. On this $\operatorname{BIBD}$, we will consider $\operatorname{AR}(m)$ correlation structures with necessarily $2 m<k=5$ (see Section 2.2). We chose $m=2$. Let be two distinct treatments $j$ and $j^{\prime}$ in $\llbracket 1,5 \rrbracket=\llbracket 1, v \rrbracket$ and $\ell, \ell^{\prime} \in \llbracket 1,2 \rrbracket=\llbracket 1, m \rrbracket$.

To verify Identity (26), we have to consider $s \in \llbracket 1,2 \rrbracket$. For $s=1$, we count in $(a)$ that $N_{d, j, j^{\prime}}^{1}=4=\omega(k-s)$, the number of times that $j$ and $j^{\prime}$ are applied consecutively to the same patient. For $s=2$, we count $N_{d, j, j^{\prime}}^{2}=3=\omega(k-s)$, the number of time that $j$ and $j^{\prime}$ are applied to the same patient at distance $s=2$. Hence, as in our proposition, the BIBD (a) is a NN2-balanced with $N_{d, j, j^{\prime}}^{s}=\omega(k-s)$ for each $s \in \llbracket 1, m \rrbracket$.

To verify 27], we have to count the value $\phi_{d, j, j^{\prime}}^{\ell *}$ which is the number of times that the treatments $j$ and $j^{\prime}$ occur together and $j$ or $j^{\prime}$ are applied to the $\ell$ th period or the $(k-\ell+1)$ th period. For example, for $\ell=1$, we have $k-\ell+1=5$; the treatment $j=2$ appears 2 times in the first period (patients $i=2$ and $i=7$ ) and also 2 times in the last period. We find that $j$ appears $4=\omega(k-1)$ times in the first or the last period. In the same way, any treatment $j^{\prime}$ appears 4 times in the first or the last period. Then $\phi_{d, j, j^{\prime}}^{1 *}=8=2 \omega(k-1)$ as in (27). We count also that $\phi_{d, j, j^{\prime}}^{2 *}=8$ and Identity ( $\left.i i i\right)$ of Optimality Theorem holds.

Now, for Equality (28), consider the value $\phi_{d, j, i}^{\ell}$; i.e. the number of times that the treatment $j$ is applied to the patient $i$ at the period $\ell$ or $(k-\ell+1)$. When $\ell=\ell^{\prime}$, the value $\sum_{i=1}^{10} \phi_{d, j, i}^{\ell} \phi_{d, j^{\prime}, i}^{\ell^{\prime}}$ is the number of times that $j$ and $j^{\prime}$ appear together at the periods $\ell$ and $k-\ell+1$. By definition of SB of strength 2 , this value is the index $\omega=1$ of (a) (that we can verify on the design itself). When $\ell \neq \ell^{\prime}$, by symmetry, we can chose $\ell=1$ and $\ell^{\prime}=2$. For example, $j=1$ and $j^{\prime}=5$ appear together 2 times in the same line of (a) with $j$ in period $\ell=1$ or 5 and $j^{\prime}$ is in period $\ell^{\prime}=2$ or 4 (patients $i=1$ and $i=9$ ). It is the same for any $j, j^{\prime}$ with $j \neq j^{\prime}$. Then $\sum_{i=1}^{10} \phi_{d, j, i}^{\ell} \phi_{d, j^{\prime}, i}^{\ell^{\prime}}=2=2 \omega$ and as in our proposition $\sum_{i=1}^{10} \phi_{d, j, i}^{\ell} \phi_{d, j^{\prime}, i}^{\ell^{\prime}}=\omega\left(2-\delta_{\ell, \ell^{\prime}}\right)$. Then $(i v)$ of Optimality Theorem holds.

Two finish with Equality [29], suppose that $\ell \neq \ell^{\prime}$. Then necessarily $\left|\ell-\ell^{\prime}\right|=1$ and we can chose $\ell=1$ and $\ell^{\prime}=2$. The value $N_{d, j, j^{\prime}, i}^{\mid \ell-\ell^{\prime}}=1$ if the treatments $j$ and $j^{\prime}$ are applied consecutively to the patient $i$ and else $N_{d, j, j^{\prime}, i}^{\left|\ell-\ell^{\prime}\right|}=0$. For example, $j=1$ and $j^{\prime}=5$ appear $\omega=1$ times together in periods $\ell=1$ and $\ell^{\prime}=2$ (patient $i=10$ ) and $\omega=1$ times in periods $k-\ell+1=5$ and $k-\ell^{\prime}+1=4$ (patient $i=8$ ). It is the same for any $j, j^{\prime}, j \neq j^{\prime}$. 
Then, as in our proposition 21, we obtain

$$
\sum_{i=1}^{10} N_{d, j, j^{\prime}, i}^{s}\left(\phi_{d, j, i}^{\ell} \phi_{d, j^{\prime}, i}^{\ell^{\prime}}+\phi_{d, j^{\prime}, i}^{\ell} \phi_{d, j, i}^{\ell^{\prime}}\right)=2=2 \omega\left(\ell, \ell^{\prime} \in \llbracket 1,2 \rrbracket, s=\left|\ell-\ell^{\prime}\right|=1\right)
$$

and Identity $(v)$ of Optimality Theorem holds.

In consequence, by applying Optimality Theorem 5.1 or Proposition 21, we can conclude that the design $(a)$ is universally optimal.

\section{PROOFS}

\subsection{Proof of Lemma7,}

We consider the matrix $\mathbb{M}=\left(\gamma_{\ell, \ell^{\prime}}\right)_{1 \leq \ell, \ell^{\prime} \leq k}$ and we search to express the sum $p_{\ell}=$ $\sum_{\ell^{\prime}=1}^{k} \gamma_{\ell, \ell^{\prime}}$ of the elements of the line $\ell$ in the form given in the Lemma what we search to prove. By symmetry of matrix $\mathbb{M}$ we can suppose that $\ell \in \llbracket 1, k-m \rrbracket$.

We will proceed in two steps. Let us first calculate the portion $\alpha_{\ell}$ of this sum for $\ell^{\prime} \geq \ell$ and then we calculate the rest $\beta_{\ell}$. With the sum $p_{\ell}=\alpha_{\ell}+\beta_{\ell}$, we will find the appropriate formula.

First compute $\alpha_{\ell}=\sum_{\ell^{\prime}=\ell}^{k} \gamma_{\ell, \ell^{\prime}}$.

From Formula (8) of Lemma 5, we have the following expression of each $\gamma_{\ell, \ell^{\prime}}$ for $\ell^{\prime} \in$ $\llbracket \ell, k \rrbracket:$

$$
\gamma_{\ell, \ell+s}=\sum_{u=0}^{l-1} \theta_{u} \theta_{u+s} \quad \text { for } s \in \llbracket 0, k-\ell \rrbracket
$$

Then

(31) $\alpha_{\ell}=\sum_{s=0}^{k-\ell} \sum_{u=0}^{\ell-1} \theta_{u} \theta_{u+s}=\sum_{u=0}^{\ell-1} \theta_{u} \sum_{s=0}^{k-\ell} \theta_{u+s}=\sum_{u=0}^{\ell-1} \theta_{u} \sum_{b=u}^{k+u-\ell} \theta_{b}=\sum_{u=0}^{\ell-1} \theta_{u} \sum_{b=u}^{m} \theta_{b}$.

Because $\theta_{b}=0$ for each $b>m$ and for each $u \in \llbracket 0, \ell-1 \rrbracket$ we have $k+u-\ell \geq k-\ell \geq$ $k-(k-m)=m$.

Now Compute $\beta_{\ell}=\sum_{\ell^{\prime}=1}^{\ell-1} \gamma_{\ell, \ell^{\prime}}=\sum_{\ell^{\prime}=1}^{\ell-1} \gamma_{\ell^{\prime}, \ell}$, by symmetry. We have:

$$
\beta_{\ell}=\sum_{\ell^{\prime}=1}^{\ell-1} \sum_{u=0}^{\ell^{\prime}-1} \theta_{u} \theta_{u+\left(\ell-\ell^{\prime}\right)}=\sum_{a=1}^{\ell-1} \theta_{a} \sum_{b=0}^{a-1} \theta_{b}
$$


To see this last identity, the reader can follow this method: write by column each element $\theta_{u} \theta_{u+\left(\ell-\ell^{\prime}\right)}$ forming the sum $\sum_{u=0}^{\ell^{\prime}-1} \theta_{u} \theta_{u+\left(\ell-\ell^{\prime}\right)}=\gamma_{\ell^{\prime}, \ell}$; the first column for $\ell^{\prime}=1$, the second for $\ell^{\prime}=2$ and so on; the elements under the diagonal are all zero; then by the summation of the elements in each same parallel line over the diagonal we find $\theta_{a} \sum_{b=0}^{a-1} \theta_{b}$ if the parallel is at distance $\ell-1-a$ of the diagonal. There are $\ell-2$ parallels. By example, the sum of the elements of the diagonal is $\theta_{\ell-1}\left(\theta_{0}+\theta_{1}+\cdots+\theta_{\ell-2}\right)$ (i.e. $a=\ell-1$ and the distance is 0$)$, the sum of the parallel just over the diagonal is $\theta_{\ell-2}\left(\theta_{0}+\theta_{1}+\cdots+\theta_{\ell-3}\right)$ (i.e. $v=\ell-2$ and the distance is 0 ); the last parallel is reduced to the only one element $\theta_{1} \theta_{0}$ (i.e. $a=1$ and the distance is $\ell-2$ ).

We have finished because from (31) and (32), we obtain the announced identity in Lemma 7 .

$$
p_{\ell}=\alpha_{\ell}+\beta_{\ell}=\sum_{u=0}^{\ell-1} \theta_{u} \sum_{b=0}^{m} \theta_{b}=a_{0}\left(a_{0}-a_{\ell}\right)
$$

with $a_{\ell}=\sum_{b=\ell}^{m} \theta_{b}$ for $\ell \in \llbracket 1, m \rrbracket$ and $a_{\ell}=0$ for $\ell>m$. In particular, for $\ell \in$ $\llbracket m+1, k-m \rrbracket$, the identity becomes $p_{\ell}=p_{m+1}=a_{0}^{2}=\left(1-\theta_{1}-\cdots-\theta_{m}\right)^{2}$.

For the last lines, as $\mathbb{M}$ is symmetric with respect to its second diagonal, $p_{\ell}=p_{k-\ell+1}$ for $\ell \in \llbracket k-m+1, k \rrbracket$.

\subsection{Proof of Lemma 12,}

We introduce some tools before addressing the proof of Lemma 12 . For $r \in \llbracket 1, k \rrbracket, \boldsymbol{e}_{r}=$ $\left(\boldsymbol{e}_{r, s}\right)_{1 \leq s \leq k}$ means the $r$-th canonical vector of $\mathbb{R}^{k}$, i.e. $\boldsymbol{e}_{r, s}=\delta_{r, s}$ (where $\delta$ is the Kronecker symbol). Note that each element of a $k \times k$-matrix $A$ is expressed in the form $A_{r, s}=$ $\boldsymbol{e}_{r}^{\prime} A \boldsymbol{e}_{s}$.

For each treatment $j \in \llbracket 1, v \rrbracket$, the $j$ th column vector $\mathbf{t}_{j}(i)$ of the matrix $T_{i}$ defined in $(7)$ is expressed as follows: for each patient $i \in \llbracket 1, b \rrbracket$, we set

$$
\mathbf{t}_{j}(i)= \begin{cases}\boldsymbol{e}_{\ell} & \text { if } j \text { is applied to } i \text { at the } \ell \text {-th period where } \ell \in \llbracket 1, k \rrbracket \\ \mathbf{0}_{k} & \text { otherwise. }\end{cases}
$$

Hence, by general notation, for each period $\ell \in \llbracket 1, k-m \rrbracket$, we have

$$
\phi_{d, j}^{\ell}= \begin{cases}\#\left\{i: \mathbf{t}_{j}(i) \in\left\{\boldsymbol{e}_{\ell}, \boldsymbol{e}_{k-\ell+1}\right\}\right\} & \text { if } \ell \in \llbracket 1, m \rrbracket \\ \#\left\{i: \mathbf{t}_{j}(i)=\boldsymbol{e}_{\ell}\right\} & \text { if } \ell \in \llbracket m+1, k-m \rrbracket .\end{cases}
$$

Remark 24. Exactly $r$ vectors $\mathbf{t}_{j}(i)$ are nonzero because exactly $r$ patients receive the treatment $j$.

Remark 25. As the considered designs are proper, each patient $i$ receives at most one time the same treatment $j$; by consequence, for each $\ell \in \llbracket 1, m \rrbracket$ and because $\ell \neq k-\ell+1$ 
when $k>2 m$, we have:

$$
\left\{i: \mathbf{t}_{j}(i)=\boldsymbol{e}_{\ell}\right\} \cap\left\{i: \mathbf{t}_{j}(i)=\boldsymbol{e}_{k-\ell+1}\right\}=\phi .
$$

We fix two distinct treatments $j, j^{\prime}$ in $\llbracket 1, v \rrbracket$ and we seek identities on the elements $\sigma^{2} C_{d, j, j}$ and $\sigma^{2} C_{d, j, j^{\prime}}$ of the matrix

$$
\sigma^{2} C_{d}=\sum_{i=1}^{b} T_{i}^{\prime} \mathbb{M} T_{i}-\sum_{i=1}^{b} T_{i}^{\prime}\left(\mathbf{1}_{k}^{\prime} \mathbb{M} \mathbf{1}_{k}\right)^{-1} \mathbb{M} \mathbf{1}_{k} \mathbf{1}_{k}^{\prime} \mathbb{M} T_{i}
$$

where $\mathbb{M}=\sigma^{2} V^{-1}$ (see Identity $(15)$ ), by considering independently the two terms in the right side of identity (35) respectively in Sections 7.2.1 and 7.2.2. We will conclude in Section 7.2.3 Also in the following the reference to the design $d$ in index will be omit.

\subsubsection{The term $\sum_{i=1}^{b} T_{i}^{\prime} \mathbb{M} T_{i}$ where $T_{i}=\left(\boldsymbol{t}_{1}(i), \ldots, \boldsymbol{t}_{v}(i)\right)$.}

The contribution of $\sum_{i=1}^{b} T_{i}^{\prime} \mathbb{M} T_{i}$ to the diagonal element $\sigma^{2} C_{j, j}$ is the value $\tau=\sum_{i=1}^{b} \tau_{i}$ where $\tau_{i}=\mathbf{t}_{j}^{\prime}(i) \mathbb{M} \mathbf{t}_{j}(i)$. From definition (33) of vectors $\mathbf{t}_{j}(i)$, we have for each patient $i$ :

$$
\tau_{i}=\sum_{\ell=1}^{k} \sum_{\left\{i: \mathbf{t}_{j}(i)=\boldsymbol{e}_{\ell}\right\}} \boldsymbol{e}_{\ell}^{\prime} \mathbb{M} \boldsymbol{e}_{\ell}=\sum_{\ell=1}^{m} \phi_{j}^{\ell} \boldsymbol{e}_{\ell}^{\prime} \mathbb{M} \boldsymbol{e}_{\ell}+\sum_{\ell=m+1}^{k-m} \phi_{j}^{\ell} \boldsymbol{e}_{\ell}^{\prime} \mathbb{M} \boldsymbol{e}_{\ell}
$$

Combining the above identity (36) and Lemma 5 applied to elements $\gamma_{\ell, \ell}=\boldsymbol{e}_{\ell}^{\prime} \mathbb{M} \boldsymbol{e}_{\ell}$ of the matrix $\mathbb{M}$ defined in Lemma 5 , we obtain (recall that $\theta_{0}=-1$ ):

$$
\begin{aligned}
\tau= & \sum_{\ell=1}^{m} \phi_{j}^{\ell}\left(\theta_{0}^{2}+\theta_{1}^{2} \cdots+\theta_{\ell-1}^{2}\right)+\sum_{\ell=m+1}^{k-m} \phi_{j}^{\ell}\left(\theta_{0}^{2}+\theta_{1}^{2} \cdots+\theta_{m}^{2}\right) \\
= & \phi_{j}^{1} \theta_{0}^{2}+\phi_{j}^{2}\left(\theta_{0}^{2}+\theta_{1}^{2}\right)+\cdots+\phi_{j}^{m}\left(\theta_{0}^{2}+\theta_{1}^{2}+\cdots+\theta_{m-1}^{2}\right) \\
& \quad+\sum_{\ell=m+1}^{k-m} \phi_{j}^{\ell}\left(\theta_{0}^{2}+\theta_{1}^{2}+\cdots+\theta_{m}^{2}\right) \\
= & \theta_{0}^{2} \sum_{\ell=1}^{k-m} \phi_{j}^{\ell}+\theta_{1}^{2} \sum_{\ell=2}^{k-m} \phi_{j}^{\ell}+\theta_{2}^{2} \sum_{\ell=3}^{k-m} \phi_{j}^{\ell}+\cdots+\theta_{m}^{2} \sum_{\ell=m+1}^{k-m} \phi_{j}^{\ell} .
\end{aligned}
$$

As $\sum_{l=1}^{k-m} \phi_{d, j}^{\ell}=r$ (see Identity (17)), we get: 


$$
\begin{aligned}
\tau & =\theta_{0}^{2} r+\theta_{1}^{2}\left(r-\phi_{j}^{1}\right)+\theta_{2}^{2}\left(r-\left(\phi_{j}^{1}+\phi_{j}^{2}\right)\right)+\cdots+\theta_{m}^{2}\left(r-\left(\phi_{j}^{1}+\phi_{j}^{2}+\cdots+\phi_{j}^{m}\right)\right) \\
& =r \sum_{u=0}^{m} \theta_{u}^{2}-\phi_{j}^{1} \sum_{u=1}^{m} \theta_{u}^{2}-\phi_{j}^{2} \sum_{u=2}^{m} \theta_{u}^{2}-\cdots-\phi_{j}^{\ell} \sum_{u=\ell}^{m} \theta_{u}^{2}-\cdots-\phi_{j}^{m} \theta_{m}^{2}
\end{aligned}
$$

Finally, the contribution $\tau$ of the term $\sum_{i=1}^{b} T_{i}^{\prime} \mathbb{M} T_{i}$ to the element $\sigma^{2} C_{j, j}$ is

$$
\tau=r b_{0}-\phi_{j}^{1} b_{1}-\phi_{j}^{2} b_{2}-\cdots-\phi_{j}^{m} b_{m}
$$

with $b_{\ell}=\sum_{u=\ell}^{m} \theta_{u}^{2}$ for $\ell \in \llbracket 1, m \rrbracket$, as defined in Lemma 12

In the same manner of the contribution of the term $\sum_{i=1}^{b} T_{i}^{\prime} \mathbb{M} T_{i}$ to the diagonal elements, we now focus us on its contribution $\mu$ to the extra-diagonal element $\sigma^{2} C_{j, j^{\prime}}$ where $\mu=$ $\sum_{i=1}^{b} \mu_{i}$ with $\mu_{i}=\mathbf{t}_{j}^{\prime}(i) \mathbb{M} \mathbf{t}_{j^{\prime}}(i)$. For this purpose, we need to introduce the following new notation: for $\ell, \ell^{\prime} \in \llbracket 1, k \rrbracket$, we denote by $\phi_{j, j^{\prime}}^{\ell, \ell^{\prime}}$ the number of patients which receive the distinct treatments $j$ and $j^{\prime}$ at the periods $\ell, \ell^{\prime}$, i.e.

$$
\phi_{j, j^{\prime}}^{\ell, \ell^{\prime}}=\#\left\{i \in \llbracket 1, b \rrbracket: \mathbf{t}_{j}(i)+\mathbf{t}_{j^{\prime}}(i)=\boldsymbol{e}_{\ell}+\boldsymbol{e}_{\ell^{\prime}}\right\} .
$$

Note that if $\ell^{\prime}=\ell$ then $\mathbf{t}_{j}(i)+\mathbf{t}_{j^{\prime}}(i) \neq \boldsymbol{e}_{\ell}+\boldsymbol{e}_{\ell^{\prime}}$ for each patient $i$ because the distinct treatments $j$ and $j^{\prime}$ can not be applied simultaneously to the same patient $i$ at the same period $\ell$. Hence we can write for $s \in \llbracket 1, k-1 \rrbracket$ :

$$
N_{j, j^{\prime}}^{s}=\sum \phi_{j, j^{\prime}}^{\ell, \ell^{\prime}}
$$

where the sum concerns all the distinct periods $\ell, \ell^{\prime}$ in $\llbracket 1, k \rrbracket$ and $s=\left|\ell-\ell^{\prime}\right| \neq 0$.

By definition of vectors $\mathbf{t}_{j}(i)$, the value $\mu_{i}$ can be not equals zero only if $\mathbf{t}_{j}(i)+\mathbf{t}_{j^{\prime}}(i)=$ $\boldsymbol{e}_{\ell}+\boldsymbol{e}_{\ell^{\prime}}$ for some periods $\ell^{\prime}, \ell$ which are necessary distinct. Moreover, as the matrix $\mathbb{M}$ is symmetric, when the identity $\mathbf{t}_{j}(i)+\mathbf{t}_{j^{\prime}}(i)=\boldsymbol{e}_{\ell}+\boldsymbol{e}_{\ell^{\prime}}$ holds, we can suppose that $\mathbf{t}_{j}(i)=\boldsymbol{e}_{\ell}$ and $\mathbf{t}_{j^{\prime}}(i)=\boldsymbol{e}_{\ell^{\prime}}$ with $\ell<\ell^{\prime}$.

Hence, by putting $u_{i j}=\mathbf{t}_{j}(i)+\mathbf{t}_{j^{\prime}}(i), u_{\ell \ell^{\prime}}=\boldsymbol{e}_{\ell}+\boldsymbol{e}_{\ell^{\prime}}$ and considering the element $\gamma_{\ell, \ell^{\prime}}=$ $\boldsymbol{e}_{\ell}^{\prime} \mathbb{M} \boldsymbol{e}_{\ell^{\prime}}$ of the matrix $\mathbb{M}$, we obtain:

$$
\mu=\sum_{1 \leq \ell<\ell^{\prime} \leq k} \sum_{\left\{i: u_{i j}=u_{\ell \ell^{\prime}}\right\}} \boldsymbol{e}_{\ell}^{\prime} \mathbb{M} \boldsymbol{e}_{\ell^{\prime}}=\sum_{1 \leq \ell<\ell^{\prime} \leq k} \gamma_{\ell, \ell^{\prime}} \phi_{j, j^{\prime}}^{\ell, \ell^{\prime}}=\sum_{\ell^{\prime}=2}^{k} \sum_{\ell=1}^{\ell^{\prime}-1} \gamma_{\ell, \ell^{\prime}} \phi_{j, j^{\prime}}^{\ell, \ell^{\prime}}
$$


For sake of clarity in the rest of this proof, we put $\phi^{\ell, \ell^{\prime}}=\phi_{j, j^{\prime}}^{\ell, \ell^{\prime}}$. We introduce in the expression of $\mu$ the values of the elements $\gamma_{\ell, \ell^{\prime}}$ of the matrix $\mathbb{M}$ given in Lemma 5. By summing the identical factors of each of the respective values $\theta_{\ell}$ and $\theta_{\ell} \theta_{\ell^{\prime}}$, we obtain:

$$
\begin{aligned}
\mu= & -\theta_{1}\left(\phi^{1,2}+\cdots+\phi^{k-1, k}\right)-\cdots-\theta_{s}\left(\phi^{\ell, \ell+s}+\phi^{\ell+1, \ell+s+1}+\cdots+\phi^{k-s, k}\right)-\cdots \\
& \quad-\theta_{m}\left(\phi_{j, j^{\prime}}^{1, m+1}+\cdots+\phi^{k-m, k}\right)+ \\
& \quad \sum_{s=1}^{m-1} \theta_{1} \theta_{1+s}\left(\phi^{2,2+s}+\cdots+\phi^{k-s-1, k-1}\right)+\sum_{s=1}^{m-2} \theta_{2} \theta_{2+s}\left(\phi^{3,3+s}+\cdots+\phi^{k-s-2, k-2}\right) \\
& +\cdots+\sum_{s=1}^{m-u} \theta_{u} \theta_{u+s}\left(\phi^{u+1, u+1+s}+\phi^{u+2, u+2+s}+\cdots+\phi^{k-s-u, k-u}\right)+\cdots \\
& +\sum_{s=1}^{2} \theta_{m-2} \theta_{m-2+s}\left(\phi^{m-1, m-1+s}+\cdots+\phi^{k-s-(m-2), k-(m-2)}\right) \\
& +\theta_{m-1} \theta_{m}\left(\phi^{m, m+1}+\cdots+\phi^{k-m, k-m+1}\right) .
\end{aligned}
$$

Recall that identity (40) says that $N_{j, j^{\prime}}^{s}=\phi^{1,1+s}+\phi^{2,2+s}+\cdots+\phi^{k-s-1, k-1}+\phi^{k-s, k}$. Putting

$$
U_{t, s}=\phi^{t, t+s}+\phi^{k-t-s+1, k-t+1}
$$

for $s \in \llbracket 1, m-1 \rrbracket$ and $t \in \llbracket 1, m-s \rrbracket$, the expression 41$]$ of $\mu$ becomes:

$$
\begin{aligned}
\mu=\sum_{s=1}^{m} \theta_{0} \theta_{s} N_{j, j^{\prime}}^{s} & +\sum_{s=1}^{m-1} \theta_{1} \theta_{1+s}\left(N_{j, j^{\prime}}^{s}-\left(U_{1, s}\right)\right) \\
& +\sum_{s=1}^{m-2} \theta_{2} \theta_{2+s}\left(N_{j, j^{\prime}}^{s}-\left(U_{1, s}+U_{2, s}\right)\right)+\cdots \\
& +\sum_{s=1}^{m-u} \theta_{u} \theta_{u+s}\left(N_{j, j^{\prime}}^{s}-\left(U_{1, s}+U_{2, s}+\cdots+U_{u, s}\right)\right)+\cdots \\
& +\sum_{s=1}^{2} \theta_{m-2} \theta_{m-2+s}\left(N_{j, j^{\prime}}^{s}-\left(U_{1, s}+U_{2, s}+\cdots+U_{m-2, s}\right)\right) \\
& +\theta_{m-1} \theta_{m}\left(N_{j, j^{\prime}}^{1}-\left(U_{1,1}+U_{2,1}+\cdots+U_{m-1,1}\right)\right)
\end{aligned}
$$


Collecting the factors of each $N_{j, j^{\prime}}^{s}$ and the factors of each $U_{t, s}$, we obtain:

$$
\mu=\sum_{s=1}^{m} N_{j, j^{\prime}}^{s} \sum_{u=0}^{m-s} \theta_{u} \theta_{u+s}-\sum_{s=1}^{m-1} \sum_{t=1}^{m-s} U_{t, s} \sum_{u=t}^{m-s} \theta_{u} \theta_{u+s}
$$

Indeed, for each $s \in \llbracket 1, m-1 \rrbracket$ and $t \in \llbracket 1, m-s \rrbracket$, the component $\beta_{t, s}$ of $\mu$ which collects the terms $U_{t, s} \theta_{a, b}$ is the following:

$$
\beta_{t, s}=-U_{t, s}\left(\theta_{t} \theta_{t+s}+\theta_{t+1} \theta_{t+1+s}+\cdots+\theta_{m-s} \theta_{m}\right) .
$$

In addition, the double summation $\sum_{s=1}^{m-1} \sum_{t=1}^{m-s} \beta_{t, s}$ collects all the terms of the form $U_{t, s} \theta_{a, b}$ in the right side of Identity (42). In order to complete the determination of $\mu$, note that:

Remark 26. Considering Identity (39) about $\phi_{j, j^{\prime}}^{\ell, \ell^{\prime}}$ and Remark 11 about the quantities $N_{j, j^{\prime}, i}^{s}$ and $\phi_{j, i}^{\ell}$, we find:

$$
\begin{aligned}
U_{t, s} & =\phi_{j, j^{\prime}}^{t, t+s}+\phi_{i, j^{\prime}}^{k-t-s+1, k-t+1} \\
& =\#\left\{i: \mathbf{t}_{j}(i)+\mathbf{t}_{j^{\prime}}(i) \in\left\{\boldsymbol{e}_{t}+\boldsymbol{e}_{t+s}, \boldsymbol{e}_{k-t+1}+\boldsymbol{e}_{k-(t+s)+1}\right\}\right\} \\
& =\sum_{i=1}^{b} N_{j, j^{\prime}, i}^{s}\left(\phi_{j, i}^{t} \phi_{j^{\prime}, i}^{t+s}+\phi_{j^{\prime}, i}^{t} \phi_{j, i}^{t+s}\right) .
\end{aligned}
$$

Finally, the contribution $\mu$ of the term $\sum_{i=1}^{b} T_{i}^{\prime} \mathbb{M} T_{i}$ to the element $\sigma^{2} C_{j, j^{\prime}}$ is:

$$
\begin{aligned}
& \mu= \sum_{s=1}^{m} N_{j, j^{\prime}}^{s} \sum_{u=0}^{m-s} \theta_{u} \theta_{u+s}- \\
& \text { (43) } \quad \sum_{s=1}^{m-1} \sum_{t=1}^{m-s}\left(\theta_{t} \theta_{t+s}+\theta_{t+1} \theta_{t+1+s}+\cdots+\theta_{m-s} \theta_{m}\right) \sum_{i=1}^{b} N_{j, j^{\prime}, i}^{s}\left(\phi_{j, i}^{t} \phi_{j^{\prime}, i}^{t+s}+\phi_{j^{\prime}, i}^{t} \phi_{j, i}^{t+s}\right) .
\end{aligned}
$$

\subsubsection{Term $\sum_{i=1}^{b} T_{i}^{\prime}\left(\mathbf{1}_{k}^{\prime} \mathbb{M} \boldsymbol{1}_{k}\right)^{-1} \mathbb{M} \boldsymbol{1}_{k} \mathbf{1}_{k}^{\prime} \mathbb{M} T_{i}$ on the right side of Identity (35).}

Note that the constant value $c=\mathbf{1}_{k}^{\prime} \mathbb{M} \mathbf{1}_{k}$ in this summation is the sum of all elements of $\mathbb{M}$ given in Equation (11). So in the sequel we consider the term $c^{-1} \sum_{i=1}^{b} T_{i}^{\prime} \mathbb{M} \mathbf{1}_{k} \mathbf{1}_{k}^{\prime} \mathbb{M} T_{i}$.

Introduce the following notation for each treatment $j_{1}$ and each patient $i$ :

$$
k_{j_{1}, i}=\mathbf{t}_{j_{1}}^{\prime}(i) \mathbb{M} \mathbf{1}_{k}
$$

As $k_{i, j_{1}}$ is a scalar and $\mathbb{M}=\mathbb{M}^{\prime}$ (i.e. $\mathbb{M}$ is symmetric), we also have:

$$
k_{j_{1}, i}=\mathbf{1}_{k}^{\prime} \mathbb{M} \mathbf{t}_{j_{1}}^{\prime}(i)
$$


Then the contribution of $c^{-1} \sum_{i=1}^{b} T_{i}^{\prime} \mathbb{M} \mathbf{1}_{k} \mathbf{1}_{k}^{\prime} \mathbb{M} T_{i}$ to the element $\sigma^{2} C_{j_{1}, j_{2}}$ for two treatments $j_{1}, j_{2}$ not necessary distincts is

$$
\omega_{j_{1}, j_{2}}=c^{-1} \sum_{i=1}^{b} k_{j_{1}, i} k_{j_{2}, i}
$$

Still with the aim of finding identities on the elements $C_{d, j, j}$ and $C_{d, j, j^{\prime}}$ of the matrix $C$, we must determine the quantity $k_{j, i}=\mathbf{t}_{j}^{\prime}(i) \mathbb{M} \mathbf{1}$.

When the treatment $j$ is not applied to the $i$ th patient, $k_{j, i}=0$ because $\mathbf{t}_{j}(i)=\mathbf{0}_{k}$. Otherwise, it is applied only once, at some $\ell$ th period and we have $k_{j, i}=\mathbf{t}_{j}^{\prime}(i) \mathbb{M} \mathbf{1}=\sum_{\ell^{\prime}=1}^{k} \gamma_{\ell, \ell^{\prime}}$.

Recall that the sum of elements of the line $\ell$ in matrix $\mathbb{M}$ is given in Lemma 7 ; for each $\ell \in \llbracket 1, k-m \rrbracket$, the value $p_{\ell}=\sum_{\ell^{\prime}=1}^{k} \gamma_{\ell, \ell^{\prime}}=a_{0}\left(a_{0}-a_{\ell}\right)$ (with $a_{\ell}=\sum_{u=\ell}^{m} \theta_{u}$ for $\ell \in \llbracket 1, m \rrbracket$ and $a_{\ell}=0$ for $\ell>m$ ) and $p_{\ell}=p_{k-\ell+1}$ for $\ell \in \llbracket k-m, k \rrbracket$. Remark that $p_{\ell}=p_{m+1}=a_{0}^{2}$ for all $\ell>m$. Thus $\forall \ell \in \llbracket 1, m \rrbracket \cup \llbracket k-m+1, k \rrbracket$ :

$$
p_{\ell}-p_{m+1}=-a_{0} a_{\ell}
$$

Now, let's determine the values of $n_{j, i}$ and $\phi_{j, i}^{\ell}$ for all $\ell \in \llbracket 1, m \rrbracket$ that we have defined above. Recall that $\mathbf{t}_{j}(i)=\boldsymbol{e}_{\ell}$ if the treatment $j$ is applied to the patient $i$ at the $\ell$-th period and $\mathbf{t}_{j}(i)=\mathbf{0}_{k}$ otherwise.

Case $\mathbf{t}_{j}(i)=\mathbf{0}_{k}: n_{j, i}=\phi_{j, i}^{1}=\cdots=\phi_{j, i}^{m}=0$ because the patient $i$ does not receive the treatment $j$.

Case $\mathbf{t}_{j}(i)=\boldsymbol{e}_{\ell}$ where $\ell \in \llbracket 1, m \rrbracket \cup \llbracket k-m+1, k \rrbracket: n_{j, i}=\phi_{j, i}^{\ell}=1$ and $\phi_{j, i}^{1}=\cdots=\phi_{j, i}^{\ell-1}=\phi_{j, i}^{\ell+1}=\cdots=\phi_{j, i}^{m}=0$.

Case $\mathbf{t}_{j}(i)=\boldsymbol{e}_{\ell}$ where $\ell \in \llbracket m+1, k-m \rrbracket: n_{j, i}=1$ and $\phi_{j, i}^{1}=\cdots=\phi_{j, i}^{m}=0$.

If the treatment $j$ is applied to the patient $i$ at one $\ell$ th period for $\ell \in \llbracket 1, k \rrbracket$ then $k_{j, i}=p_{\ell}$. Otherwise, if the treatment $j$ is not applied to the patient $i$ then $k_{j, i}=0$. By consequence, we can express the quantity $k_{j, i}$ in the following form

$$
\begin{gathered}
k_{j, i}=p_{m+1} n_{j, i}+\phi_{j, i}^{1}\left(p_{1}-p_{m+1}\right)+\phi_{j, i}^{2}\left(p_{2}-p_{m+1}\right)+\cdots \\
+\phi_{j, i}^{m}\left(p_{m}-p_{m+1}\right) .
\end{gathered}
$$

By Formulas (10) and (45), we deduce that: 


$$
\begin{aligned}
k_{j, i} & =a_{0}\left(a_{0} n_{j, i}-a_{1} \phi_{j, i}^{1}-a_{2} \phi_{j, i}^{2}-\cdots-a_{m} \phi_{j, i}^{m}\right) \\
& =a_{0}\left(a_{0} n_{j, i}-\sum_{\ell=1}^{m} a_{\ell} \phi_{j, i}^{\ell}\right) .
\end{aligned}
$$

Recall that the contribution $\sum_{i=1}^{b} T_{i}^{\prime}\left(\mathbf{1}_{k}^{\prime} \mathbb{M} \mathbf{1}_{k}\right)^{-1} \mathbb{M} \mathbf{1}_{k} \mathbf{1}_{k}^{\prime} \mathbb{M} T_{i}$ to element $\sigma^{2} C_{j, j}$ is $\omega_{j, j}=$ $c^{-1} \sum_{i} k_{j, i}^{2}$ (see (44)). By Identity (47), we have:

$$
k_{j, i}^{2}=a_{0}^{2}\left\{a_{0}^{2} n_{j, i}^{2}+\sum_{\ell=1}^{m} a_{\ell}^{2} \phi_{j, i}^{\ell}-2 a_{0} \sum_{\ell=1}^{m} a_{\ell} n_{j, i} \phi_{j, i}^{\ell}\right\}
$$

because $\left(\phi_{j, i}^{\ell}\right)^{2}=\phi_{j, i}^{\ell} \forall \ell \in \llbracket 1, m \rrbracket$, and when $\ell \neq \ell^{\prime}, \phi_{j, i}^{\ell} \phi_{j, i}^{\ell^{\prime}}=0$. By the facts

$$
\phi_{j}^{\ell}=\sum_{i=1}^{b} \phi_{j, i}^{\ell}=\sum_{i=1}^{b} n_{j, i} \phi_{j, i}^{\ell} \quad \text { and } \quad r=\sum_{i=1}^{b} n_{j, i}^{2},
$$

we finally obtain:

$$
\begin{aligned}
c w_{j, j}=\sum_{i=1}^{b} k_{j, i}^{2} & =a_{0}^{2}\left\{a_{0}^{2} r+\sum_{\ell=1}^{m} a_{\ell}^{2} \phi_{j}^{\ell}-2 a_{0} \sum_{\ell=1}^{m} a_{\ell} \phi_{j}^{\ell}\right\} \\
& =-a_{0}^{2}\left\{a_{0}\left(a_{0}-2 a_{0}\right) r-\sum_{\ell=1}^{m} \phi_{j}^{\ell} a_{\ell}\left(a_{\ell}-2 a_{0}\right)\right\}
\end{aligned}
$$

Now we search to determine the contribution $\omega_{j, j^{\prime}}=c^{-1} \sum_{i=1}^{b} k_{j, i} k_{j^{\prime}, i}, j \neq j^{\prime}$, to the element $\sigma^{2} C_{j, j^{\prime}}$. By Identity 46, we have:

$$
\begin{aligned}
k_{j, i} k_{j^{\prime}, i}= & a_{0}^{2}\left(a_{0} n_{j, i}-\sum_{\ell=1}^{m} a_{\ell} \phi_{j, i}^{\ell}\right)\left(a_{0} n_{j^{\prime}, i}-\sum_{\ell^{\prime}=1}^{m} a_{\ell^{\prime}} \phi_{j^{\prime}, i}^{\ell^{\prime}}\right) \\
= & a_{0}^{2}\left\{a_{0}^{2} n_{d, j, i} n_{j^{\prime}, i}-a_{0}\left(\sum_{\ell=1}^{m} a_{\ell} n_{j, i} \phi_{j^{\prime}, i}^{\ell}+\sum_{\ell=1}^{m} a_{\ell} n_{j^{\prime}, i} \phi_{j, i}^{\ell}\right)\right. \\
& \left.\sum_{\ell=1}^{m} \sum_{\ell^{\prime}=1}^{m} a_{\ell} a_{\ell^{\prime}} \phi_{j, i}^{\ell} \phi_{j^{\prime}, i}^{\ell^{\prime}}\right\} .
\end{aligned}
$$

By the facts $\lambda_{j, j^{\prime}}=\sum_{i=1}^{b} n_{j, i} n_{j^{\prime}, i}$ and

$$
\phi_{j, j^{\prime}}^{\ell *}=\sum_{i=1}^{b}\left(n_{j^{\prime}, i} \phi_{j, i}^{\ell}+n_{j, i} \phi_{j^{\prime}, i}^{\ell}\right) \quad \text { for all } \ell \in \llbracket 1, m \rrbracket
$$


we finally obtain:

$$
\begin{aligned}
c \omega_{j, j^{\prime}} & =\sum_{i=1}^{b} k_{j, i} k_{j^{\prime}, i} \\
& =a_{0}^{4} \lambda_{j, j^{\prime}}-a_{0}^{3} \sum_{\ell=1}^{m} a_{\ell} \phi_{j, j^{\prime}}^{\ell *}+a_{0}^{2} \sum_{\ell=1}^{m} \sum_{\ell^{\prime}=1}^{m} a_{\ell} a_{\ell^{\prime}} \sum_{i=1}^{b} \phi_{j, i}^{\ell} \phi_{j^{\prime}, i}^{\ell^{\prime}} .
\end{aligned}
$$

\subsubsection{Conclusion of the proof of Lemma 12 .}

From (35), we have:

$$
C_{j, j}=\tau-\omega_{j, j} \quad \text { and } \quad C_{j, j^{\prime}}=\mu-\omega_{j, j^{\prime}} \quad .
$$

where $\tau$ and $\mu$ come from the term $\sum_{i=1}^{b} T_{i}^{\prime} \mathbb{M} T_{i}$ studied in Section 7.2.1 and $\omega_{j, j}$ and $\omega_{j, j^{\prime}}$ come from the term $\sum_{i=1}^{b} T_{i}^{\prime}\left(\mathbf{1}_{k}^{\prime} \mathbb{M} \mathbf{1}_{k}\right)^{-1} \mathbb{M} \mathbf{1}_{k} \mathbf{1}_{k}^{\prime} \mathbb{M} T_{i}$ studied in Section 7.2.2

It suffice to collect the values of $\tau, \mu, \omega_{j, i}$ and $\omega_{j, j^{\prime}}$ from Identities (38), 43), (48) and (50) to conclude that our Lemma 12 is true.

\subsection{Proof of Lemma 14.}

Firstly, as $d$ is a BIBD, we have $\beta=\sum_{j=1}^{v} \sum_{j^{\prime} \neq j} \lambda_{d, j, j^{\prime}}=\sum_{j=1}^{v} \sum_{j^{\prime} \neq j} \lambda=v(v-1) \lambda$ Secondly, we count $\beta$ in this different manner:

$$
\beta=\sum_{j=1}^{v} \sum_{j^{\prime} \neq j} \lambda_{d, j, j^{\prime}}=b k(k-1)
$$

because there are $b$ patients and exactly $k(k-1)$ distinct pairs of treatments by patient (recall that $k \leq v$ ) The identification of the two expressions of $\beta$ prove the searched identity satisfied by $\lambda$ :

$$
\lambda=\lambda_{d, j, j^{\prime}}=\frac{b k(k-1)}{v(v-1)} \quad \forall j, j^{\prime} \in \llbracket 1, v \rrbracket, j \neq j^{\prime}
$$

\subsection{Proof of Lemma 15 .}

We suppose that the design $d$ is $\mathrm{NN} m$-balanced. Then $N_{d, j, j^{\prime}}^{s}$ is a constant $N_{d}^{s}$ that we intend to establish.

We fix $s \in \llbracket 1, m \rrbracket$. We compute with to manner the sum $\alpha=\sum_{j=1}^{v} \sum_{j^{\prime} \neq j} N_{d, j, j^{\prime}}^{s}$. Firstly, as the design is $\mathrm{NN} m$-balanced, each $N_{d, j, j^{\prime}}^{s}$ equals a constant $N_{d}^{s}$ which does not depend 
on the choice $j, j^{\prime}$. Then we have:

$$
\alpha=\sum_{j=1}^{v} \sum_{j^{\prime} \neq j} N_{d}^{s}=v(v-1) N_{d}^{s}
$$

Secondly, we fixe one treatment $j$ and suppose that a patient $i$ receives this treatment. Recall that $j$ is administered no more than once to a same patient. For the patient $i$, there exist $2=\sum_{j^{\prime} \neq j} N_{d, j, j^{\prime}, i}^{s}$ treatments at distance $s$ to $j$ if $j$ is not applied in the first $s$ or the last $s$ periods (i.e.when $\sum_{\ell=1}^{s} \phi_{d, j, i}^{\ell}=0$ ), otherwise $\phi_{d, j, i}^{\ell}=1$ for (only) one period $\ell \in \llbracket 1, s \rrbracket\left(\right.$ i.e.when $\sum_{\ell=1}^{s} \phi_{d, j, i}^{\ell}=1$ ) and there exists only $1=\sum_{j^{\prime} \neq j} N_{d, j, j^{\prime}, i}^{s}$ treatment at distance $s$ to $j$. Then

$$
\sum_{j^{\prime} \neq j} N_{d, j, j^{\prime}, i}^{s}=2-\sum_{\ell=1}^{s} \phi_{d, j, i}^{\ell}
$$

Moreover $j$ appears exactly $r$ times in $d$. Then by considering firstly all patients $i$,

$$
\sum_{j^{\prime} \neq j} N_{d, j, j^{\prime}}^{s}=2 r-\sum_{\ell=1}^{s} \phi_{d, j}^{\ell}
$$

and secondly Identity (21), we obtain the second expression of $\alpha$ :

$$
\alpha=\sum_{j=1}^{v} \sum_{j^{\prime} \neq j} N_{d, j, j^{\prime}}^{s}=\sum_{j=1}^{v}\left(2 r-\sum_{\ell=1}^{s} \phi_{d, j}^{\ell}\right)=2 r v-2 b s
$$

As $r v=k b$ (see (4)), the identification of our two expressions of $\alpha$ implies the searched identity satisfied by $N_{d}^{s}$ :

$$
N_{d}^{s}=N_{d, j, j^{\prime}}^{s}=\frac{2 b(k-s)}{v(v-1)} \quad \forall j, j^{\prime} \in \llbracket 1, v \rrbracket, j \neq j^{\prime} .
$$

\subsection{Proof of Lemma 16.}

Let be an $\mathrm{NN} m$-balanced design $d$ with $k=v$ (i.e. the number of periods equals the number treatments). We have also $r=b$ because $r v=k b$.

We search to prove that for each $\ell \in \llbracket 1, m \rrbracket$ the quantities $\phi_{d, j}^{\ell}$ and $\phi_{d, j, j^{\prime}}^{\ell *}$ are independent of distinct treatments $j, j^{\prime}$ and to express them without $j$ and $j^{\prime}$.

Let be $s \in \llbracket 1, m \rrbracket$. As $d$ is an NNm-balanced design, from $(i i)$ of Lemma 15 the quantity $N_{d, j, j^{\prime}}^{s}$ is the constant $N_{d}^{s}=2 b(k-s) / v(v-1)=2 b(v-s) / v(v-1)$ since $k=v$.

From (52) and since $N_{d, j, j^{\prime}}^{s}$ is the constant $N_{d}^{s}$ for all $s \in \llbracket 1, m \rrbracket$, we have:

$$
\sum_{\ell=1}^{s} \phi_{d, j}^{\ell}=2 r-(v-1) N_{d}^{s}
$$


As $r=b$, the previus equality becomes $\sum_{\ell=1}^{s} \phi_{d, j}^{\ell}=\frac{2 b s}{v}$. Then we conclude that for each $s \in \llbracket 1, m \rrbracket:$

$$
\phi_{d, j}^{s}=\sum_{\ell=1}^{s} \phi_{d, j}^{\ell}-\sum_{\ell=1}^{s-1} \phi_{d, j}^{\ell}=\frac{2 b}{v}
$$

which is the identity expected by Lemma 16 .

Now since $k=v$ and each treatment is administered at most once for each patient, each patient $i$ receives each of the $v$ distinct treatments once and only once. That means $n_{d, j, i}=$ 1 for all $j \in \llbracket 1, v \rrbracket$. Then Identity (49] becomes

$$
\phi_{d, j, j^{\prime}}^{\ell *}=\phi_{d, j}^{\ell}+\phi_{d, j^{\prime}}^{\ell}=\frac{4 b}{v} \quad \forall \ell \in \llbracket 1, m \rrbracket
$$

and the Lemma is proved.

\subsection{Proof of Theorem 5.1 .}

By the consequence of Lemma 12 (see remark 13), all the competitor designs have the same trace. Hence, by Proposition 10, the universal optimality of a design $d$ for the model $\operatorname{AR}(m)$ is satisfied when the information matrix $C_{d}$ of $\widehat{\gamma}$ is completely symmetric; that is to say that its extra-diagonal elements $C_{d, j, j^{\prime}}$ are all independent of $j, j^{\prime}\left(j \neq j^{\prime}\right)$ because the sum by line (and by column) of $C_{d}$ is null (see (16)). According to hypothesis of Theorem 5.1. we will prove that each of the five terms of $C_{d, j, j^{\prime}}$ in Lemma 12 is independent to $j, j^{\prime}$.

As the design is $\mathrm{NN} m$-balanced $\operatorname{BIBD}(v, b, r, k, \lambda)$, Identities $(i)$ and $(i i)$ of Lemmas 14 and 15 imply that two of the terms of $C_{d, j, j^{\prime}}$ are independent to $j, j^{\prime}$. If Identities $(i i i),(i v)$ and $(v)$ of Theorem 5.1 hold then the three others terms of $C_{d, j, j^{\prime}}$ are independent to $j$ and $j^{\prime}$.

Remark 27. Note that, for a better presentation of Identity $(v)$ in Theorem 5.1, we have replace the values $s, t$ appearing in $C_{d, j, j^{\prime}}$ by the values $\ell, \ell^{\prime}$. This is possible as explained below. Suppose that Identity $(v)$ holds for each $\ell$ and $\ell^{\prime}$ such that $\ell \neq \ell^{\prime} \in \llbracket 1, m \rrbracket$. Then, by the symmetry in $\ell, \ell^{\prime}$ of the expression $\alpha_{\ell, \ell^{\prime}}=N_{j, j^{\prime}, i}^{\left|\ell-\ell^{\prime}\right|}\left(\phi_{j, i}^{\ell} \phi_{j^{\prime}, i}^{\ell^{\prime}}+\phi_{j^{\prime}, i}^{\ell} \phi_{j, i}^{\ell^{\prime}}\right)$, we can suppose that $\ell<\ell^{\prime}$ and we have $\alpha_{\ell, \ell^{\prime}}=N_{j, j^{\prime}, i}^{\left|\ell-\ell^{\prime}\right|}\left(\phi_{j, i}^{\ell} \phi_{j^{\prime}, i}^{\ell+\left|\ell-\ell^{\prime}\right|}+\phi_{j^{\prime}, i}^{\ell} \phi_{j, i}^{\ell+\left|\ell-\ell^{\prime}\right|}\right)$. Then

$$
\alpha_{\ell, \ell^{\prime}}=N_{j, j^{\prime}, i}^{s}\left(\phi_{j, i}^{t} \phi_{j^{\prime}, i}^{t+s}+\phi_{j^{\prime}, i}^{t} \phi_{j, i}^{t+s}\right)
$$

with $s=\left|\ell-\ell^{\prime}\right| \in \llbracket 1, m-1 \rrbracket$ and $\ell=t \in \llbracket 1, m-s \rrbracket$ as expected in the summation in the expression of $C_{d, j, j^{\prime}}$. Conversely, let $s \in \llbracket 1, m-1 \rrbracket$ and $t \in \llbracket 1, m-s \rrbracket$ as in the summation in $C_{d, j, j^{\prime}}$. Then the two distinct periods $\ell=t$ and $\ell^{\prime}=t+s$ in $\llbracket 1, m \rrbracket$ verify Identity (56). 
In the following, we will prove that Identities $(i i i),(i v)$ and $(v)$ of Theorem 5.1 are coherent. More precisely, for each identity, we will suppose that the term in the left side is a constant and we prove that it equals to the right side.

7.6.1. Identity $($ iii). For each treatment $j$, we first need to establish the following identity:

$$
\sum_{j^{\prime} \neq j} \phi_{d, j, j^{\prime}}^{\ell *}=(k-2) \phi_{d, j}^{\ell}+2 r
$$

Proof. We develop $\sum_{j^{\prime} \neq j} \phi_{j, j^{\prime}}^{\ell *}$

$$
\sum_{j^{\prime} \neq j} \phi_{j, j^{\prime}}^{\ell *}=\sum_{j^{\prime} \neq j} \sum_{i=1}^{b}\left(n_{j^{\prime}, i} \phi_{j, i}^{\ell}+n_{j, i} \phi_{j^{\prime}, i}^{\ell}\right)=\sum_{i=1}^{b} \phi_{j, i}^{\ell} \sum_{j^{\prime} \neq j} n_{j^{\prime}, i}+\sum_{i=1}^{b} n_{j, i} \sum_{j^{\prime} \neq j} \phi_{j^{\prime}, i}^{\ell}
$$

The first term of the right hand of the previous identity is

$$
\alpha=\sum_{i=1}^{b} \phi_{j, i}^{\ell} \sum_{j^{\prime} \neq j} n_{j^{\prime}, i}=\sum_{i=1}^{b} \phi_{j, i}^{\ell} \sum_{j=1}^{v} n_{j^{\prime}, i}-\sum_{i=1}^{b} \phi_{j, i}^{\ell} n_{j, i}=\phi_{j}^{\ell} k-\phi_{j}^{\ell}
$$

by definition of $\phi_{j}^{\ell}$ and since each patient $i$ receive $k$ treatments. The second term is

$$
\beta=\sum_{i=1}^{b} n_{j, i} \sum_{j^{\prime} \neq j} \phi_{j^{\prime}, i}^{\ell}=\sum_{i=1}^{b} n_{j, i} \sum_{j^{\prime}=1}^{v} \phi_{j^{\prime}, i}^{\ell}-\sum_{i=1}^{b} n_{j, i} \phi_{j, i}^{\ell}=r \times 2-\phi_{j}^{\ell}
$$

because $d$ is equireplicated (i.e. $j$ appears $r$ times in $d$ ) and only 2 treatments $j^{\prime}$ can be applied to a same patient $i$ at the $\ell$ th and the $(k-\ell+1)$ th periods (i.e. $\phi_{j^{\prime}, i}^{\ell}=1$ and not 0 for this two treatments). By the sum of $\alpha$ and $\beta$, Identity (57) is proved.

From Formulas (57) and (21), we obtain finally:

$$
\sum_{j=1}^{v} \sum_{j^{\prime} \neq j} \phi_{d, j, j^{\prime}}^{\ell *}=2 b(k-2)+2 r v=2 b(k-2)+2 b k=4 b(k-1)
$$

because $r v=b k$. Suppose that each $\phi_{d, j, j^{\prime}}^{\ell *}$ is independent to $j, j^{\prime}$. Then $\sum_{j=1}^{v} \sum_{j^{\prime} \neq j} \phi_{d, j, j^{\prime}}^{\ell *}=$ $v(v-1) \phi_{d j, i, j^{\prime}}^{\ell *}$ Thus from 58 , we obtain Identity

$$
\text { (iii) } \phi_{d, j, j^{\prime}}^{\ell *}=\frac{4 b(k-1)}{v(v-1)}
$$


7.6.2. Identity $(i v)$. Consider two distinct periods $\ell$ and $\ell^{\prime}$ and set a patient $i$. Four distinct treatments $j_{1}, \ldots, j_{4}$ are applied to this patient at the respective periods $\ell, k-\ell+1, \ell^{\prime}, k-$ $\ell^{\prime}+1$. Then $\phi_{d, j_{1}, i}^{\ell}=\phi_{d, j_{2}, i}^{\ell}=\phi_{d, j_{3}, i}^{\ell^{\prime}}=\phi_{d, j_{4}, i}^{\ell^{\prime}}=1$ and the other values are zero; by consequence:

$$
\sum_{j=1}^{v} \sum_{j^{\prime} \neq j} \phi_{d, j, i}^{\ell} \phi_{d, j^{\prime}, i}^{\ell^{\prime}}=\phi_{d, j_{1}, i}^{\ell}\left(\phi_{d, j_{3}, i}^{\ell^{\prime}}+\phi_{d, j_{4}, i}^{\ell^{\prime}}\right)+\phi_{d, j_{2}, i}^{\ell}\left(\phi_{d, j_{3}, i}^{\ell^{\prime}}+\phi_{d, j_{4}, i}^{\ell^{\prime}}\right)=4
$$

and

$$
\sum_{j=1}^{v} \sum_{j^{\prime} \neq j} \phi_{d, j, i}^{\ell} \phi_{d, j^{\prime}, i}^{\ell}=\phi_{d, j_{1}, i}^{\ell} \phi_{d, j_{2}, i}^{\ell}+\phi_{d, j_{2}, i}^{\ell} \phi_{d, j_{1}, i}^{\ell}=2
$$

If the quantity $\sum_{i=1}^{b} \phi_{d, j, i}^{\ell} \phi_{d, j^{\prime}, i}^{\ell^{\prime}}$ is independent of $j, j^{\prime}$ then, by the same reasoning as for (iii), we find:

$$
\text { (iv) } \sum_{i=1}^{b} \phi_{d, j, i}^{\ell} \phi_{d, j^{\prime}, i}^{\ell^{\prime}}=\frac{b\left(2+2\left(1-\delta_{\ell, \ell^{\prime}}\right)\right)}{v(v-1)} \text { for all } \ell, \ell^{\prime} \in \llbracket 1, m \rrbracket
$$

where $\delta_{\ell, \ell^{\prime}}$ is the Kronecker symbol.

7.6.3. Identity $(v)$. We fix $s \in \llbracket 1, m-1 \rrbracket$ and $t \in \llbracket 1, m-s \rrbracket$. By the same reasoning as above, for a patient $i$, four distinct treatments $j_{1}, \ldots, j_{4}$ are applied at the respective distinct periods $t, k-t+1, t+s, k-(t+s)+1$. Then

$$
\begin{aligned}
\beta_{t, s}=\sum_{j=1}^{v} \sum_{j^{\prime} \neq j}\left(\phi_{d, j, i}^{t} \phi_{d, j^{\prime}, i}^{t+s}+\phi_{d, j^{\prime}, i}^{v} \phi_{d, j, i}^{t+s}\right) & =\sum_{j=1}^{v} \phi_{d, j, i}^{t} \sum_{j^{\prime} \neq j} \phi_{d, j^{\prime}, i}^{t+s}+\sum_{j=1}^{v} \phi_{d, j, i}^{t+s} \sum_{j^{\prime} \neq j} \phi_{d, j^{\prime}, i}^{t} \\
& =2\left(\phi_{d, j_{1}, i}^{t}+\phi_{d, j_{2}, i}^{t}\right)\left(\phi_{d, j_{3}, i}^{t+s}+\phi_{d, j_{4}, i}^{t+s}\right) \\
& =8 .
\end{aligned}
$$

But, in this sum, we have count 4 situations in which two treatments among $j_{1}, \ldots, j_{4}$ are applied at distance $s$ and 4 situations in which two treatments among $j_{1}, \ldots, j_{4}$ are applied at distance $\geq m>s$ because $k>2 m$. For the firsts 4 situations, we have $N_{d, j, j^{\prime}, i}^{s}=1$ for the 4 others we have $N_{d, j, j^{\prime}, i}^{s}=0$. Then

$$
\begin{aligned}
\sum_{j=1}^{v} \sum_{j^{\prime} \neq j} N_{d, j, j^{\prime}, i}^{s}\left(\phi_{d, j, i}^{t} \phi_{d, j^{\prime}, i}^{t+s}+\phi_{d, j^{\prime}, i}^{t} \phi_{d, j, i}^{t+s}\right) & =\sum_{j=1}^{v} \sum_{j^{\prime} \neq j} N_{d, j, j^{\prime}, i}^{s}\left(\phi_{d, j, i}^{t} \phi_{d, j^{\prime}, i}^{t+s}+\phi_{d, j^{\prime}, i}^{t} \phi_{d, j, i}^{t+s}\right) \\
& =\frac{1}{2} \beta_{t, s}=4 .
\end{aligned}
$$


Hence, if each quantity $\sum_{i=1}^{b} N_{d, j, j^{\prime}, i}^{s}\left(\phi_{d, j, i}^{t} \phi_{d, j^{\prime}, i}^{t+s}+\phi_{d, j^{\prime}, i}^{t} \phi_{d, j, i}^{t+s}\right)$ is independent to $j, j^{\prime}(j \neq$ $\left.j^{\prime}\right)$, the following identity holds:

$$
\text { (v) } \sum_{i=1}^{b} N_{d, j, j^{\prime}, i}^{s}\left(\phi_{d, j, i}^{t} \phi_{d, j^{\prime}, i}^{t+s}+\phi_{d, j^{\prime}, i}^{t} \phi_{d, j, i}^{t+s}\right)=\frac{4 b}{v(v-1)} .
$$

\subsection{Proof of Theorem 5.2 ,}

From Theorem 5.1 and Lemma 16 our Theorem is proved

\section{Acknowledgments}

We thank Professor Paul Deheuvels and Professor Pierre Druilhet for their constructive suggestions which have improved the quality of this article.

\section{REFERENCES}

Azzalini, A. and Giovagnoli, A., 1987. Some optimal designs for repeated measurements with autoregressive errors. Biometrika, 74(4), 725-734.

Benchekroun, K., 1993. Association-balanced arrays with applications to experimental design. Ph.D. thesis, Dept. of Statistics, The University of North Carolina, Chapel Hill.

Chêng, C. (1988). A note on the optimality of semi balanced arrays. in : Dodge, federov, wynn (eds),. Optimal Design and Analysis of Experiment. North-Holland, Amsterdam, pages $115-122$.

Deheuvels, P. et Derzko, G. 1991. Block designs for early-stage clinical trials. Rapport technique LSTA.

Dey, A. 2010. INCOMPLET BLOCK DESIGNS. Indian Statistical Institute, New Delhi, India.

Gill, P. S. and Shukla, G. K., 1985a. Efficiency of nearest neighbour balanced block designs for correlated observations. Biometrika, 72(3), 539-544.

Gill, P. S. and Shukla, G. K., 1985b. Experimental designs and their efficiencies for spatially correlated observations in two dimensions. Comm. Statist. A-Theory Methods, 14(9), 2181-2197.

Grondona, M. O. and Cressie, N., 1993. Efficiency of block designs under stationary second-order autoregressive errors. Sankhyā: The Indian Journal of Statistics, Series A, 55(2), 267-284.

Hedayat, N.A., Sloane, J., Stufken, J., 1999. Orthogonal Arrays: Theory and Applications. Springer, New York.

Kiefer, J., 1958. On the nonrandomized optimality and randomized nonoptimality of symmetrical designs. Ann. Statist., 29, 675-699.

Kiefer, J., 1975a. Balanced block designs and generalized Youden designs. I. Construction (patchwork). Ann. Statist., 3, 109-118. 
Kiefer, J., 1975b. Construction and optimality of generalized Youden designs. in: A survey of statistical design and linear models (Proc. Internat. Sympos., Colorado State Univ., Ft. Collins, Colo., 1973), North-Holland, Amsterdam, pp. 333-353.

Kiefer, J. and Wynn, H. P., 1981. Optimum balanced block and Latin square designs for correlated observations. Ann. Statist., 9(4), 737-757.

Koné, M. and Valibouze, A., 2011. Plans en blocs incomplets pour la structure de corrélation NN $m$. Annales de l'ISUP, 55(2-3), 65-88.

Kunert, J., 1987. Neighbour balanced block designs for correlated errors. Biometrika, 74(4), 717-724.

Kunert, J.,1985. Optimal repeated measurements designs for correlated observations and analysis by weighted least squares. Biometrika, 72(2), 375-389.

Kushner, H. B., 1997. Optimal repeated measurements designs: the linear optimality equations. Ann. Statist., 25(6), 2328-2344.

Martin, R. J., 1998. Optimal designs for small-sized blocks under dependence. J. Combin. Inform. System Sci., 23(1-4), 95-124. J. N. Srivastava: felicitation volume.

Martin, R. J. and Eccleston, J. A., 1991. Optimal incomplete block designs for general dependence structures. J. Statist. Plann. Inference, 28(1), 67-81.

Morgan, J. P. and Chakravarti, I. M., 1988. Block designs for first and second order neighbor correlations. Ann. Statist., 16(3), 1206-1224.

Mukhopadhyay, A. C., 1972. Construction of BIBD's from OA's combinatorial arrangements analogous to OA's. Calcutta Statist. Assoc. Bull., 21, 45-50.

Passi, R. M., 1976. A weighting scheme for autoregressive time averages. Journal of Applied Meteorology, 15(2), 117-119.

Ramanujacharyulu, C., 1966. A new general series of balanced incomplete block designs. Proc. Amer. Math. Soc., 17, 1064-1068.

Rao, C., 1946. Hypercubes of strength 'd' leading to confounded designs in factorial experiments. Bull. Calcutta Math. Soc., 38, 67-78.

Rao, C., 1947. Factorial experiments derivable from combinatorial arrangements of arrays. J. R. S. S., Suppl, 09, 128-139.

Rao, C., 1961. Combinatorial arrangements analogous to orthogonal arrays. Sankhy $\bar{a}$ Ser. A, pp. 283-286.

Rao, C. R., 1973. Some combinatorial problems of arrays and applications to design of experiments. In: Survey of combinatorial theory (Proc. Internat. Sympos., Colorado State Univ., Ft. Collins, Colo., 1971), North-Holland, Amsterdam, pp. 349-359.

Satpati, S. K. and Parsad, R., 2004. Construction and cataloguing of nested partially balanced incomplete block designs. Ars Combin., 73, 299-309.

Satpati, S. K., Parsad, R. and Gupta, V. K., 2007. Efficient block designs for dependent observations - a computer-aided search. Comm. Statist. Theory Methods, 36(5-8), 1187-1223. 
Siddiqui, M. M., 1958. On the inversion of the sample covariance matrix in a stationary autoregressive process. Ann. Math. Statist., 29, 585-588.

Stufken, J., 1991. Some families of optimal and efficient repeated measurements designs. J. Statist. Plann. Inference., 27, 75-83.

Wei, W. W. S., 1990. Time series analysis. Univariate and multivariate methods. AddisonWesley Publishing Company Advanced Book Program, Redwood City, CA.

Wise, J., 1955. The autocorrelation function and the spectral density function. Biometrika, 42, 151-159.

Mamadou Koné, UPMC Univ Paris 06, CNRS, Laboratoire LSTA, F-75005 Paris, France. EMAIL: MADOUSAM.KONE@GMAIL.COM

Annick Valibouze, Sorbonne Université, CNRS, LiP6 \& LPSM, F-75005 Paris, France EMAIL: ANNICK.VALIBOUZE@SORBONNE-UNIVERSITE.FR 